\title{
Explorando las posibilidades de mejora de la calidad térmica y energética en aulas escolares de Costa Rica a través del uso de estrategias pasivas
}

\author{
Emily Vargas Soto
}

Artículo

Afiliación: Universidad de Costa Rica, Costa Rica.

E-mail: emily.vargas@ucr.ac.cr

Recibido: 3 de octubre del 2019

Aceptado: 7 de noviembre del 2019

\section{Emily Vargas Soto}

Doctora en Arquitectura y Urbanismo con énfasis en Hábitat Sustentable y Eficiencia Energética por la Universidad del Bío-Bío en Chile (2016) y Máster en Arquitectura y Construcción por parte de la Universidad de Costa Rica (2012). Actualmente ejerce como docente e investigadora de la Escuela de Arquitectura de la Universidad de Costa Rica y coordinadora del Laboratorio de Arquitectura Tropical y el Laboratorio de Modelado y Fabricación Digital. Realiza investigación relacionada con temas de desempeño térmico y energético de las edificaciones y la aplicación de estrategias pasivas y diseño bioclimático para el mejoramiento y adaptación climática de las construcciones en climas tropicales.

\section{Resumen}

En países tropicales en desarrollo, las consideraciones de eficiencia energética y calidad en edificaciones escolares es mínima, comprometiendo las condiciones deseables de confort interno. Este trabajo explora los efectos sobre el confort térmico y la eficiencia energética que se generan al realizar variaciones paramétricas a la configuración típica de las aulas naturalmente ventiladas en zonas tropicales de Costa Rica. Utilizando simulaciones dinámicas mediante Design Builder, datos meteorológicos y encuestas de confort, se analizaron aulas con el mismo patrón de diseño en tres localidades con tendencia a sobrecalentamiento. Se revisó la demanda energética y la variación de la temperatura operativa del recinto al adecuar parámetros bioclimáticos de enfriamiento pasivo y control solar. Los resultados indican que ciertas configuraciones pueden optimizar el rendimiento de la tipología de los casos estudiados, dando paso a buscar su aplicación dentro de los parámetros de diseño de las nuevas edificaciones a construir en zonas similares. Se demuestra el impacto de la elección de los parámetros adecuados de diseño y el uso de herramientas de simulación en la verificación del desempeño de las edificaciones.

Palabras clave: aulas escolares; eficiencia energética; estrategias pasivas; mejoramiento térmico; simulaciones dinámicas; trópico.

Exploring the possibilities of improving thermal and energy quality in school classrooms in Costa Rica through the use of passive strategies

\section{Abstract:}

In tropical developing countries, the considerations of energy efficiency and quality of school buildings are minimal, compromising the conditions of internal comfort. This paper explores the effects on thermal comfort and energy generated by performing parametric variations on the typical configuration of naturally ventilated classrooms in tropical Costa Rica. Using dynamic simulations by Design Builder, weather data, and surveys of comfort, classrooms with the same design pattern in three locations prone to overheating were analyzed. Energy demand and improving the operating temperature of the enclosure by varying bioclimatic parameters of passive cooling and solar control were reviewed. The results indicate that certain configurations can optimize the performance of the typology of cases studied. The impact of choosing appropriate design parameters and the use of simulation tools in verifying building performance is demonstrated.

Keywords: classrooms, dynamic simulations; energy efficiency; passive strategies; thermal improvement; tropics. 


\section{Introducción}

n países con climas tropicales donde las condiciones medioambientales son cálidas y húmedas, un reto para el diseño y la construcción es proponer el balance correcto entre métodos naturales de ventilación y métodos activos en el orden de disminuir el impacto económico, los costos de operación a largo plazo, el impacto cultural y lograr mayor eficiencia de los recursos (Sosa \& Siem, 2004). Los sistemas de enfriamiento son los mayores consumidores de energía en climas tropicales, así lo reflejan reportes de la Comisión Económica para América Latina y el Caribe (2009) acerca de la perspectiva energética de Latinoamérica. En esta región, el promedio de temperatura, especialmente en la época seca, sobrepasa los rangos de confort para los ocupantes establecidos originalmente por Fanger (1972) de acuerdo a las condiciones ambientales, metabólicas y de vestimenta. La satisfacción del usuario se acerca más a respuestas del modelo adaptativo, estudiado y definido por de Dear y Brager (2001) e incorporado por la ASHRAE (2013) en el ANSI/ASHRAE Standard 55-2013. Este propone el concepto de que los usuarios en edificaciones naturalmente ventiladas, utilizando sistemas pasivos, pueden sentirse confortables a temperaturas internas más altas. Al momento de aumentar la temperatura, el usuario tiene la oportunidad de operar las aperturas con el fin de aumentar o disminuir el flujo de aire, controlando las condiciones del ambiente interno (Nitatwichit, Khunatorn, Tantakitti \& Tippayawong, 2012).

Se ha comprobado que las edificaciones diseñadas bajo el concepto de ventilación natural tienen el potencial de mejorar los ambientes de trabajo si tomamos en cuenta el consumo de energía y el confort interno y lo comparamos con sistemas controlados mecánicamente (Bordass, Cohen, Standeven, \& Leaman, 2001; Givoni, 2011). Sin embargo, hay que tener en cuenta que, en climas cálidos como el tropical, es más difícil mantener condiciones internas aceptables y lograr el control de las cargas térmicas solamente utilizando ventilación natural (Rajapaksha \& Hyde, 2012; Givoni, 1994). Para esto, es necesario combinar otros aspectos relacionados con el diseño arquitectónico y los patrones de configuración espacial para mejorar los flujos internos de aire (Prianto \& Depecker, 2003).

La adaptación del usuario al estar expuesto a situaciones climáticas específicas juega un papel importante en la definición de las condiciones espaciales. La historia de exposición térmica del usuario a un ambiente define su percepción del mismo. Las edificaciones naturalmente ventiladas proveen ambientes más diversamente dinámicos, que pueden asociarse con condiciones internas más estimulantes para los sentidos del usuario, o sea con un mayor rango de tolerancia (de Dear, 2006, 2011). Un adecuado conocimiento de los aspectos que acercan el usuario al confort puede potenciar ajustes en las condiciones de control climático de las edificaciones y, dependiendo del tipo de edificación, reducir los costos de energía beneficiando la calidad del usuario.

Gobiernos de países desarrollados como China, Australia y Estados Unidos han enfocado esfuerzos en implementar medidas en edificios públicos como una manera de dar paso a estrategias que pueden mejorar la calidad espacial en sectores económicos que tiene un alto impacto en el consumo energético y en las condiciones físicas del usuario (Roaf, Nicol \& de Dear, 2013).

Edificios escolares han sido el foco de estudios varios (Corgnati, Filippi \& Viazzo, 2007; Liang, Lin \& Hwang, 2012; Wargocki \& Wyon, 2007), donde se ha demostrado el impacto que tiene el ambiente de la edificación en usuarios que deben permanecer por jornadas de entre seis y ocho horas diarias por más de doscientos días al año y con rangos de edad que requieren ambientes propicios para fomentar el aprendizaje y lograr resultados de calidad.

La importancia de la calidad física del espacio y del confort ambiental (confort térmico, ventilación, acústica, iluminación y calidad del aire) es evidente en ambientes escolares. El confort térmico y la ventilación son dos de los aspectos esenciales para determinar la calidad espacial y la salud de los estudiantes que realizan labores de aprendizaje en espacios por períodos rutinarios y extendidos. Se ha demostrado que la exposición prolongada al sobrecalentamiento y bajas renovaciones de aire pueden disminuir las capacidades analíticas y de respuesta de los estudiantes (Lin, de Dear, Matzarakis \& 
Hwang, 2009). Mantener el control de estos aspectos es vital para lograr ambientes óptimos.

En la calidad espacial de las edificaciones públicas en regiones tropicales, ha tenido influencia las respuestas tipológicas establecidas a través del tiempo y la falta de lineamientos y normativas claras para el diseño de establecimientos educacionales. Latinoamérica ha tenido un desarrollo similar de la infraestructura educativa, marcada por las inversiones gubernamentales en períodos históricos de reformas educativas con el fin de establecer políticas locales de educación y extensión de servicios dentro de los países (Walter, 2000). La estandarización constructiva a un mismo modelo de aula aumentó el número de edificaciones, pero limitó la adaptación de los espacios diseñados a las diferentes condiciones climáticas de las zonas en que fueron implantadas. Además, el uso de un sólo criterio de diseño y tecnologías foráneas resultaron en una arquitectura que eventualmente no se ha asociado con el clima y los requerimientos culturales y tecnológicos del trópico, comprometiendo el confort térmico (Sosa \& Siem, 2004).

En Costa Rica, para el 2016 que inició este estudio, un 43\% de las 4071 escuelas existentes a ese momento habían sido diseñadas en sistemas industriales prefabricados con un mismo modelo de aula típica. Para los 5 años siguientes, se había estimado un incremento del $3 \%$ de la infraestructura educativa bajo los mismos estándares constructivos. De acuerdo con el Programa Estado de la Nación en Desarrollo Humano Sostenible (2013), específicamente en el Estado de la Educación Costarricense, se evidenciaba la urgencia en buscar mejoras en la tipología actual para optimizar su rendimiento térmico y energético en las diferentes localidades donde se desarrollarían los nuevos modelos.

El $87 \%$ de las escuelas desarrolladas para ese año se encontraban fuera del área metropolitana, en la vertiente Pacífica, mayormente divididas en tres sectores centralcosteros (Península de Nicoya, Región Pacífica Central y Brunca) con altos rangos de sobrecalentamiento en época seca con temperaturas entre los $28^{\circ} \mathrm{C}$ y los $36^{\circ} \mathrm{C}$. Es posible estudiar la posibilidad de mejorar el rendimiento de dichas escuelas estableciendo la influencia que tiene la tipología actual y otras variables independientes sobre el rendimiento y las condiciones climáticas en el aula.

El objetivo de este estudio es explorar los efectos en el confort térmico y el desempeño energético generados por cambios en parámetros arquitectónicos (diseño) y configuración de las aulas típicas del trópico costarricense. Específicamente, se realiza una revisión acerca de las consecuencias de aplicar estrategias de enfriamiento pasivo (control solar, disminución y disipación de calor), demostrando la posibilidad que tienen estas técnicas para mejorar la tipología básica.

\section{Casos de Estudio: Escuelas de Bebedero, Paquita y Buenos Aires (Veracruz)}

Este estudio se referencia en la zona tropical cálido-húmeda de Costa Rica, ubicada entre la zona del Trópico de Cáncer y la línea del Ecuador. Esta área se enfrenta por lo general a las condiciones producidas por la Zona de Convergencia Intertropical (ZCIT). La ZCIT es una zona de baja presión donde se reúnen los vientos alisios del norte y el sur, masas de aire y altas temperaturas que producen abundante nubosidad y fuertes lluvias.

Costa Rica posee un clima estratificado de manera variado, influenciado por las zonas de vida (relación entre piso altitudinal, temperatura, humedad y otros factores ambientales). La principal razón de esta diversidad se produce por estar localizada en el medio de un océano (Pacífico) y un mar (Caribe), sumado a la variedad de elevaciones del territorio central dividido por la Cordillera Volcánica Central, El Valle Central, la Vertiente Pacífica y Atlántica, por mencionar algunas de las zonas con diversas condiciones, pero siempre entre el rango de microclimas tropicales. Éstos son dominados principalmente por una condición no estacional con una época lluviosa entre mayo y noviembre y una época seca con poca o ninguna lluvia durante los meses de diciembre a abril. 
Los estudios fueron realizados en seis aulas naturalmente ventiladas en tres diferentes zonas de la costa Pacífica de Costa Rica. Las edificaciones escolares están localizadas en Bebedero - Cañas (10²2'10.21" N / 85011'37.86 O con una altura de $12 \mathrm{msnm})$, Paquita - Puntarenas (09027'40.57" N / 840'50.81" O con una altura de $10 \mathrm{msnm}$ ) y Veracruz - Buenos Aires (0909'45.44" N / 84¹9'26.35" O con una altura de 385 msnm).

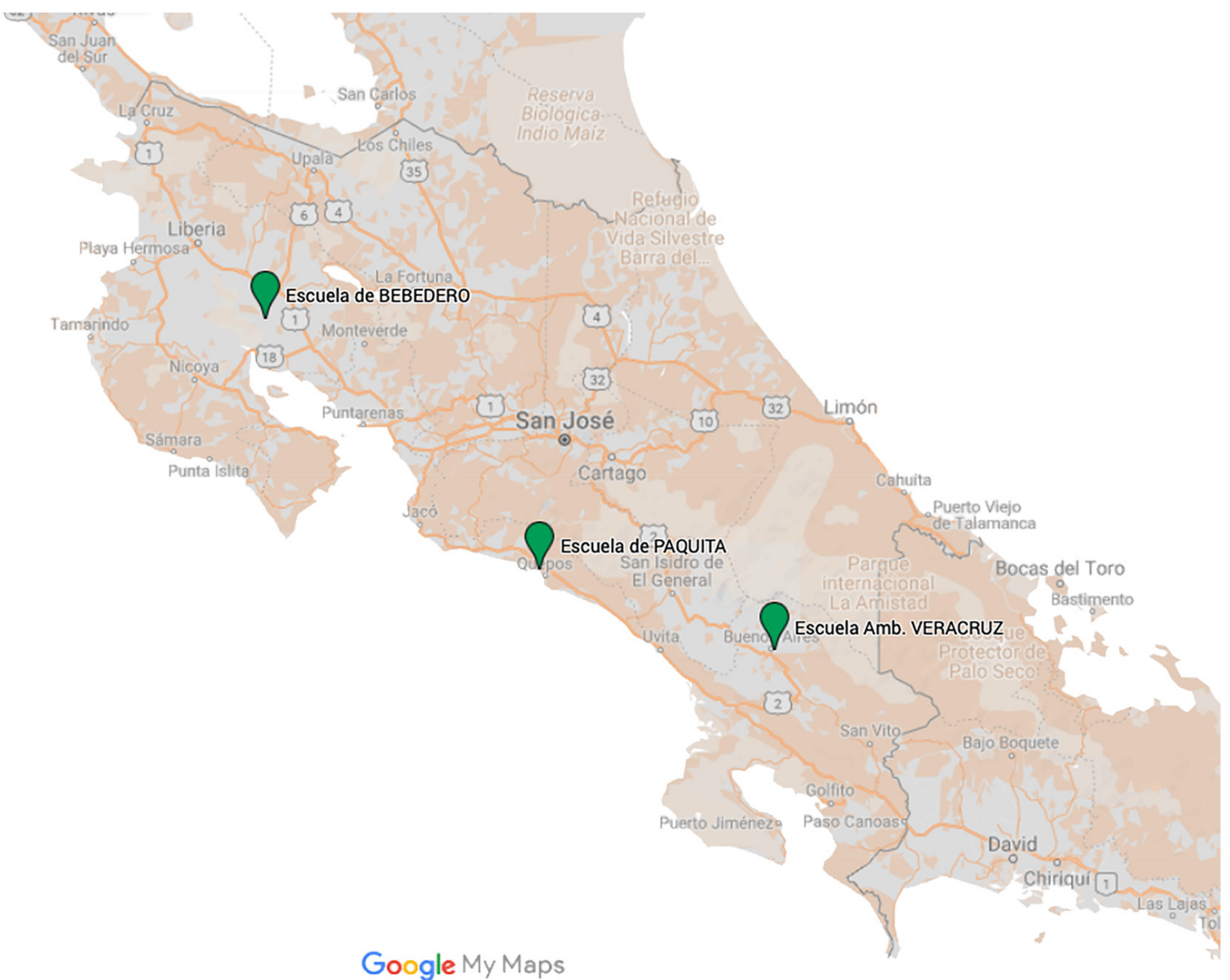

Figura 1. Localización de las escuelas utilizadas como caso de estudios. Mapa de Costa Rica desarrollado por Google Maps.

Fuente: Elaboración propia.
La Figura 1 muestra la localización de cada escuela analizada en el mapa de Costa Rica.

Los estudios de caso seleccionados permiten un escenario real para implementar la simulación como herramienta en la mejora del rendimiento, basado en casos construidos y la creación de estrategias aplicables a las nuevas edificaciones por construir en las zonas afectadas con el sobrecalentamiento.

Las zonas donde se ubican tienen rangos promedios de temperatura del aire exterior entre $25.4^{\circ} \mathrm{C}$ y $29.7^{\circ} \mathrm{C}$ con un máximo de $34.4^{\circ} \mathrm{C}$ y un mínimo de $19.1^{\circ} \mathrm{C}$, de acuerdo con los datos promedios aportados por el IMN (Instituto Meteorológico Nacional).

Las aulas de educación general básica están ocupadas principalmente por niños entre los 9 y 13 años que realizan actividades académicas. El promedio de tasa metabólica corresponde a 1.2 met de acuerdo a como lo definen la ISO 7730:1994 y la ASHRAE 55:2013. Dentro de estos espacios, se cuenta con ventanas operables en lados opuestos de fachada (ventilación cruzada) y ventiladores en cielo, que en conjunto, son los principales proveedores de ventilación natural; a esto se suma que los ocupantes cuentan con libertad para operar y con esto modificar las condiciones del ambiente interno. 
Figura 2. Planta de distribución y vista 3D del modelo de aula utilizado. Fuente: Planos de la DIEE- Ministerio de Educación de Costa Rica modificados por el autor.

Tabla 1. Resumen de áreas y materiales que componen envolvente del aula típica.

Fuente: Elaboración propia.

\section{Descripción de la tipología del aula}

Las aulas investigadas utilizan un único modelo de aula diseñada de acuerdo con el Compendio de Normas y Recomendaciones para la Construcción de Edificios Educacionales del Ministerio de Educación de Costa Rica, con una de serie de requerimientos mínimos. Las salas tienen dimensiones internas mínimas de $50.5 \mathrm{~m}^{2} \mathrm{de}$ área y entre 2.50 y $2.70 \mathrm{~m}$ de altura, construidas con un sistema de concreto prefabricado. Cada una con un máximo de ocupación de 34 estudiantes, pero con un promedio que ronda los 20 estudiantes. Se recomienda el uso de iluminación natural desde el norte, por tanto, la orientación de ventanas generalmente respeta esta condición. El mínimo tamaño de las ventanas en fachadas es equivalente a una quinta parte de la superficie total del aula, que corresponde a $10.1 \mathrm{~m}^{2}$ de área vidriada (en vidrio monolítico simple) y están protegidas por aleros externos. La Figura 2 muestra la planta de distribución y la configuración actual del aula en isométrico.

Las áreas y materiales que componen la envolvente típica del aula se resumen en la Tabla 1.
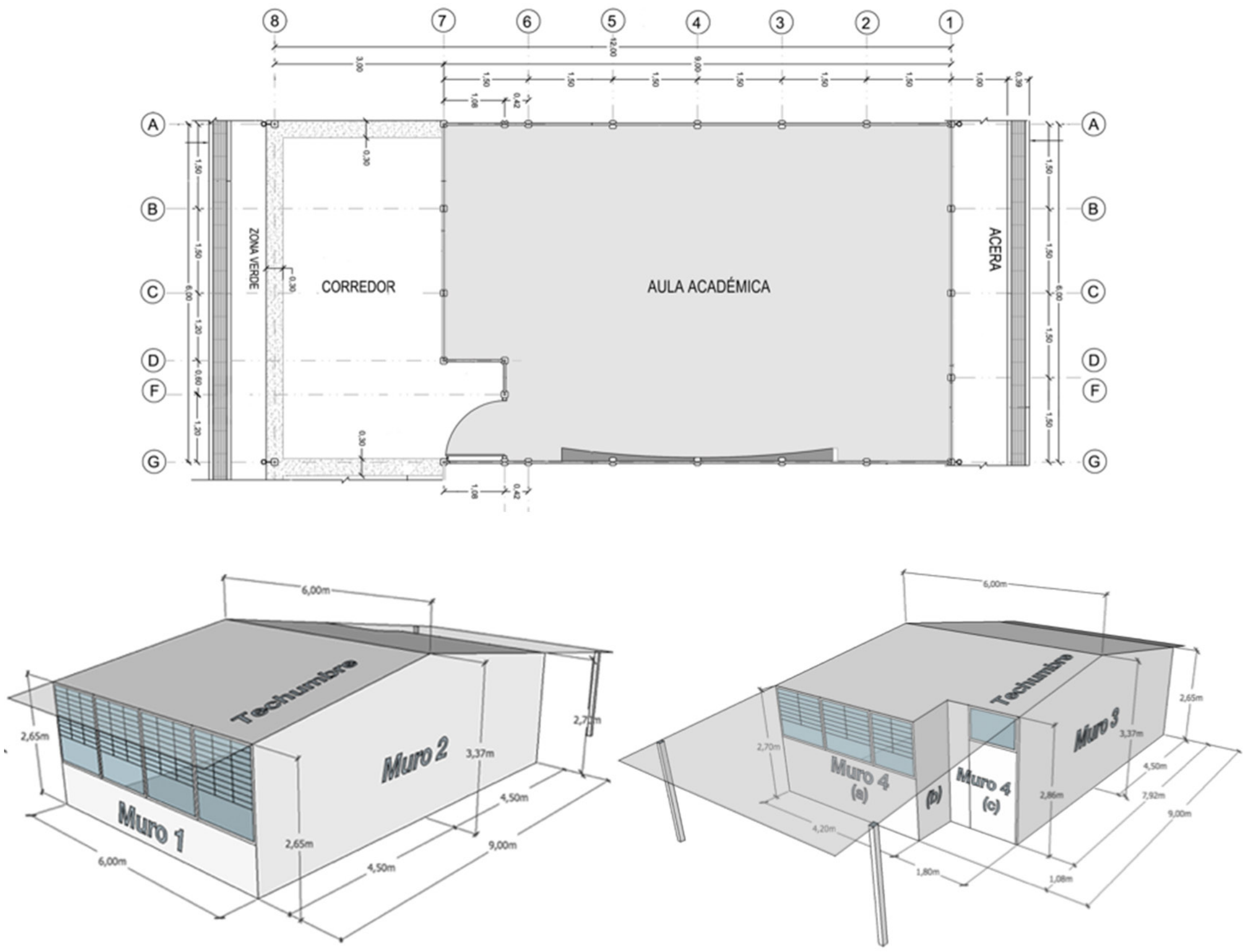

\begin{tabular}{lcl}
\hline Componente & Área & Material \\
\hline Muro 1 & $5,7 \mathrm{~m}^{2}$ & Baldosa de concreto (Concreto reforzado con 1\% de acero) y espesor de $0,038 \mathrm{~m}$ \\
Muro 2 & $27,9 \mathrm{~m}^{2}$ & \\
Muro 3 & $24,2 \mathrm{~m}^{2}$ & \\
Muro 4 (a) & $6,1 \mathrm{~m}^{2}$ & \\
Muro 4 (b) & $3 \mathrm{~m}^{2}$ & \\
Muro 4 (c) & $1,6 \mathrm{~m}^{2}$ & \\
\hline Techumbre & $83,2 \mathrm{~m}^{2}$ & Exterior en lámina de acero esmaltado blanco de 0,44mm, aislante térmico de EPS (Polietileno expandido) \\
\hline Piso & & de $5 \mathrm{~mm}$ de espesor sobre estructura metálica de hierro negro de 15cm con calibre de 1,5mm de espesor. \\
\hline
\end{tabular}




\section{Condiciones de confort térmico}

Estudios iniciales fueron realizados para reconocer las condiciones del lugar, tomar mediciones climáticas y encuestar al usuario para verificar su comportamiento ante las condiciones climáticas y establecer las condiciones de confort reales.

Las encuestas fueron realizadas de acuerdo con la ISO 10551 e ISO 7730 y aplicadas a 104 estudiantes de educación básica entre los 9 y 13 años. Se realizaron además mediciones experimentales (temperaturas de bulbo seco -interior y exterior-, humedad relativa, temperaturas de bulbo húmedo, velocidad del viento, CO2) con el fin de evaluar las condiciones internas y correlacionarlas con los modelos de confort calculados.

Los resultados con respecto a la escala de sensación térmica muestran que un $67 \%$ de los estudiantes encuestados reportaron sentirse confortables o cerca del confort (con una escala de sensación térmica entre 1 y -1) y con un promedio de temperaturas internas cerca de los $30^{\circ} \mathrm{C}$ y una humedad relativa de entre el $50 \%$ y el $62 \%$. Esto confirma lo establecido por Bravo y González (2001) en un estudio para ciudades tropicales como Maracaibo en Venezuela. Ellos encontraron que las personas que permanecen en ambientes sujetos a variación libre de la temperatura en ambientes no manipulados adquieren una mayor adaptación y tolerancia a las altas temperaturas y la humedad. Lo anterior en comparación con personas que no son de la zona o que permanecen en ambientes controlados, especialmente en climas cálidos y tropicales.

Las respuestas acerca de la sensación térmica de los 105 individuos fueron obtenidas en un rango de Tbs (Temperatura de Bulbo Seco) interna entre los $30.4^{\circ} \mathrm{C}$ y los $33.1^{\circ} \mathrm{C}$.

Los resultados de las encuestas y de las mediciones en sitio fueron comparados con cálculos del modelo adaptativo de confort para encontrar el rango de adaptación que el usuario posee en condiciones de sobrecalentamiento. El modelo de confort adaptativo fue desarrollado con el objetivo de generar índices de que tomaran en cuentala capacidad de las personas para influir en su propio sentido de confort. En este caso de estudio, la fórmula del modelo simple de confort adaptativo para edificios con funcionamiento pasivo (sin acondicionamiento mecánico) (1) desarrollada por Humphreys, Nicol y \& Raja (2007) fue aplicada.

$$
T_{-} c=12.9+\left(T_{-}(\mathrm{e}-\mathrm{ref})^{*} 0.54\right)
$$

Tabla 2. Tabla comparativa del rango de temperatura de confort para el caso de la ciudad de Bebedero

Fuente: Elaboración propia.

\begin{tabular}{|c|c|c|c|c|c|}
\hline \multicolumn{6}{|c|}{ Caso Bebedero - Época Seca (meses con tendencia a sobrecalentamiento } \\
\hline & Dic & Ene & Feb & Mar & $\mathrm{Abr}$ \\
\hline $\mathrm{T}^{0}(1)$ & & & & $30,4^{\circ} \mathrm{C}$ & $30,7^{\circ} \mathrm{C}$ \\
\hline $\mathrm{T}^{0}(2)$ & $28,4^{\circ} \mathrm{C}$ & $28,6^{\circ} \mathrm{C}$ & $29,1^{\circ} \mathrm{C}$ & $29,6^{\circ} \mathrm{C}$ & $29,7^{\circ} \mathrm{C}$ \\
\hline $\mathrm{T}(3)$ & $30,4^{\circ} \mathrm{C}$ & $30,6^{\circ} \mathrm{C}$ & $31,1^{\circ} \mathrm{C}$ & $31,7^{\circ} \mathrm{C}$ & $30,9^{\circ} \mathrm{C}$ \\
\hline $\mathrm{T}^{\mathrm{o}}(4)$ & $26,4^{\circ} \mathrm{C}$ & $26,6^{\circ} \mathrm{C}$ & $27,1^{\circ} \mathrm{C}$ & $27,7^{\circ} \mathrm{C}$ & $27,7^{\circ} \mathrm{C}$ \\
\hline
\end{tabular}

(1) Temperatura de Confort percibida por los estudiantes.

(2) Temperatura de Confort de acuerdo al modelo simple de confort adaptativo aplicado.

(3) Límite superior, Zona de Confort de acuerdo al modelo de confort adaptativo.

(4) Límite inferior, Zona de Confort de acuerdo al modelo de confort adaptativo.

La Tabla 2 muestra un ejemplo de los datos obtenidos para el caso de Bebedero con respecto al modelo simple de confort para la estación seca. La temperatura de confort percibida por el usuario, $\mathrm{T}^{\circ}(1)$, está dentro de los límites máximos y mínimos establecidos por el modelo, pero más cercano al límite superior. Estos datos se tomaron en cuenta en el proceso de simulación para evaluar el confort logrado a través del proceso de optimización. El límite inferior de confort es de alrededor de los $27^{\circ} \mathrm{C}$ para las tres ciudades. Este se estableció como la temperatura operativa a alcanzar en las simulaciones que utilizan soporte mecánico por medio de ventiladores. 


\section{Definición del modelo de simulación}

Parámetros para los modelos de simulación energética y confort.

Fueron realizadas simulaciones dinámicas para el aula típica localizada en la Costa Pacífica de Costa Rica. La demanda de energía por enfriamiento, el consumo por iluminación y la temperatura de confort para el recinto fueron considerados para la simulación. Se utilizó Design Builder como una interfaz de Energy Plus y bases de datos climáticas generadas por el software Meteonorm 7.

El flujo de trabajo de Design Builder (Figura 3) se inicia con la selección del lugar y el clima correspondiente a través de un archivo climático (.epw), seguido de la creación de la geometría del modelo por medio de la interfaz CAD integrada. Las listas de los parámetros definibles incluyen cargas internas (con los patrones de ocupación y actividades), tipo de construcción, aberturas (ventanas y puertas), la iluminación y los sistemas activos de acondicionamiento HVAC (si se aplica). Una vez que la definición de todos los parámetros de entrada se haya completado, se puede realizar el día de diseño y / o simulaciones anuales.

Figura 3. Organigrama para la definición de parámetros en Design Builder.

Fuente: Rallapalli (2010) (traducida y editada por el autor).

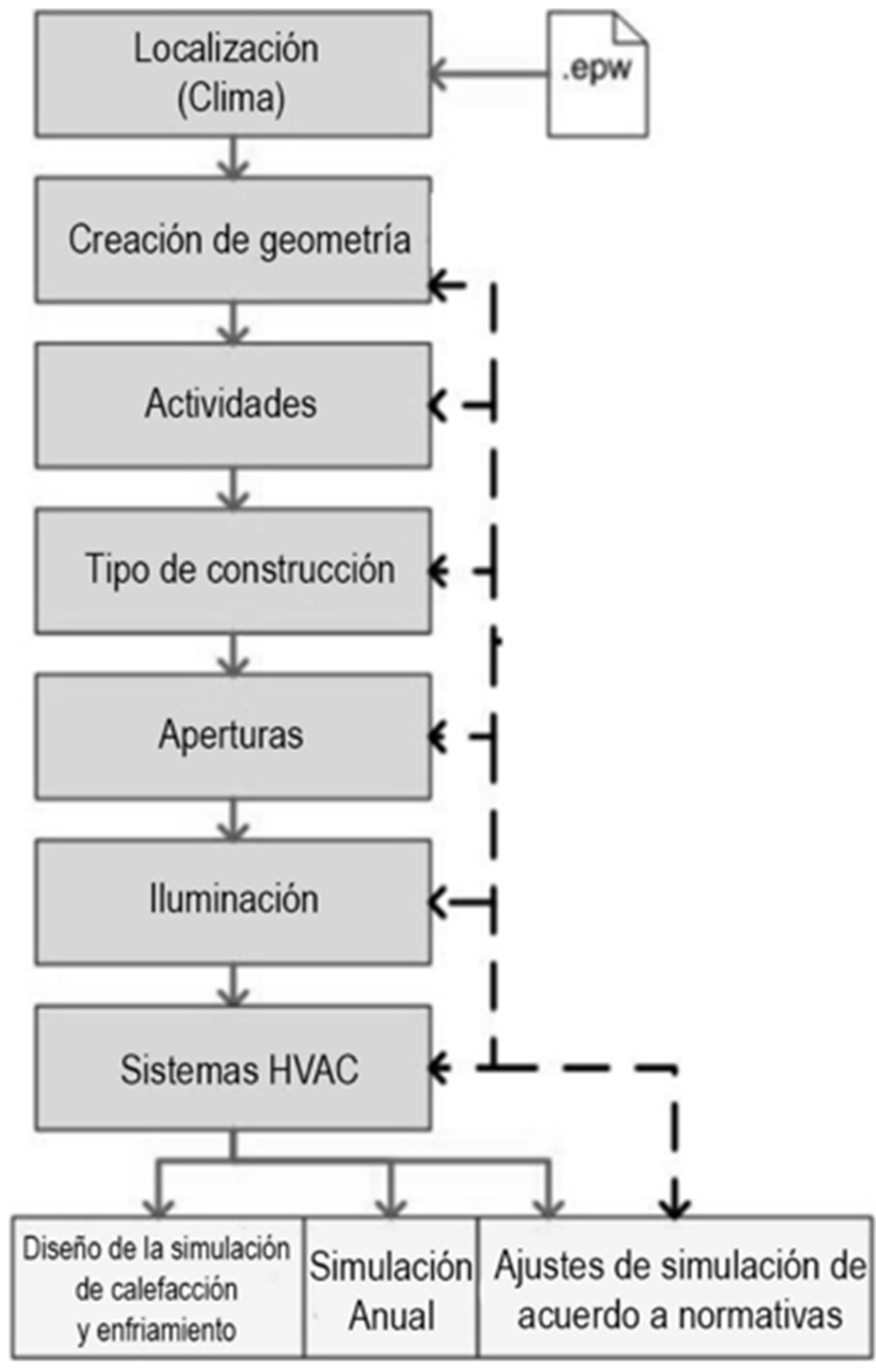

Para el proceso de la evaluación mediante simulación dinámica, tanto de los casos base como los de los casos optimizados, se generaron dos escenarios de trabajo: (1) el primero, calculando la cantidad de energía necesaria para que el ambiente interno alcance la temperatura operativa fijada (suponiendo el uso de enfriamiento activo) y (2) el segundo, con funcionamiento libre (free-running) sin equipos mecánicos y con un ambiente naturalmente ventilado, calculando así las temperaturas operativas alcanzadas en el interior del recinto.

A pesar de que las aulas funcionan sin sistemas activos de acondicionamiento, se plantea el escenario (1) con el fin de obtener un indicador comparativo. El valor de energía calculada es directamente proporcional a los datos de temperaturas operativas 
y disminuye asociado con ellas, lo que permite que en la etapa de aplicación de estrategias se identifiquen rápidamente los escenarios con mejor desempeño sin necesidad de calcular todas las temperaturas horarias asociadas. En otras palabras, al definir un "setpoint" cercano a la temperatura de confort y calcular el dato de energía requerida para enfriamiento, se observa que cuanto menor sea el valor obtenido más cercanas estarán las temperaturas operativas del recinto de alcanzar el confort definido. Los casos con menor requerimiento energético para enfriamiento cuentan con mejores condiciones internas y temperaturas operativas internas más cercanas al rango de confort térmico calculado.

\section{Calibración del modelo con los parámetros fijos.}

El modelo de simulación se calibra de acuerdo con las generalidades de diseño para las aulas definidas anteriormente. Se utilizan estos 'parámetros fijos' tanto para la simulación del caso base (rendimiento actual real) como para las soluciones de optimización planteadas en los modelos de cálculo de la demanda de energía y las condiciones de confort.

Los parámetros fijos incluyen cargas internas, modo de ventilación y la definición de características físicas. Dichas condiciones de borde se resumen en la Tabla 3.

\begin{tabular}{|c|c|c|c|}
\hline & $\begin{array}{l}\text { Caso Base } \\
\text { Bebedero }\end{array}$ & $\begin{array}{l}\text { Caso Base } \\
\text { Paquita }\end{array}$ & $\begin{array}{l}\text { Caso Base } \\
\text { Veracruz }\end{array}$ \\
\hline Épocas del año & \multicolumn{3}{|c|}{$\begin{array}{l}\text { Seca: diciembre a mayo } \\
\text { Lluviosa: junio a noviembre }\end{array}$} \\
\hline Orientación fachada ventanas exteriores & Norte & Noreste & Sureste \\
\hline Ganancias internas & \multicolumn{3}{|c|}{$\begin{array}{l}\text { Estudiantes realizando trabajo ligero } 66 \mathrm{~W} \text {, adultos caminando o en reposo } 108 \mathrm{~W} \\
\text { Iluminación artificial } 300 \text { lux }-11,25 \mathrm{~W} / \mathrm{m} 2 \\
\text { No se considera el uso de computadoras o equipo adicional en las aulas } \\
\text { Densidad ocupacional promedio: veinte (20) estudiantes por clase y un docente }(0,39 \text { personas } \\
\text { m2) }\end{array}$} \\
\hline Período de operación & \multicolumn{3}{|c|}{ Lunes a viernes, de las 8:00 a.m. a las 5 p.m. (dos jornadas) } \\
\hline Períodos no operados (sin ganancias internas) & \multicolumn{3}{|c|}{$\begin{array}{l}\text { Medio año: entre } 30 \text { de junio y } 11 \text { de julio } \\
\text { Época de vacaciones fin de año: entre } 15 \text { de diciembre y } 10 \text { de febrero }\end{array}$} \\
\hline Rango de temperaturas exteriores & $\begin{array}{l}\text { 32,4 } 4^{\circ} \mathrm{C} \text { Max. Med. Anual } \\
23,9^{\circ} \mathrm{C} \text { Min. Med. Anual }\end{array}$ & $\begin{array}{l}30,9^{\circ} \mathrm{C} \text { Max. Med. Anual } \\
22,7^{\circ} \mathrm{C} \text { Min. Med. Anual }\end{array}$ & $\begin{array}{l}31,2^{\circ} \mathrm{C} \text { Max. Med. Anual } \\
20,5^{\circ} \mathrm{C} \text { Min. Med. Anual }\end{array}$ \\
\hline Rango de confort identificado & $22-29,7^{\circ} \mathrm{C}$ & $22,1-29,9^{\circ} \mathrm{C}$ & $21,3-29^{\circ} \mathrm{C}$ \\
\hline Materiales de la envolvente & \multicolumn{3}{|c|}{$\begin{array}{l}\text { Muros y columnas en concreto prefabricado reforzado } \\
\text { Ventanas en vidrio claro de } 3 \mathrm{~mm} \\
\text { Cubierta exterior en lámina de acero galvanizada con aislamiento EPS de } 5 \mathrm{~mm} \text { sobre estructura } \\
\text { de hierro negro. }\end{array}$} \\
\hline Ventilación utilizada & \multicolumn{3}{|c|}{ Ventilación cruzada 7-8 litros/s por persona } \\
\hline Área vidriada con respecto al área de piso & \multicolumn{3}{|c|}{$30 \%(16,4 \mathrm{~m} 2)$ distribuidas en $20 \%$ ventanas operables y $10 \%$ ventanas fijas } \\
\hline
\end{tabular}

Tabla 3. Condiciones de borde utilizadas para simular los casos base de las tres localidades en estudio.

Fuente: Elaboración propia.
Como se observa en la tabla, se definieron cargas internas de personas, equipos e iluminación, períodos de ocupación, períodos libres o fuera de ocupación y rangos de horas de uso.

Las temperaturas de operación durante el período de ocupación se definieron con respecto a datos tomados y rangos de confort calculados entre 22 y 31 grados centígrados aproximadamente. Para ventilación, se realizaron simulaciones con rangos 
de $2 \mathrm{ACH}$ (cambios de aire por hora) mínimos en períodos de ocupación. También se consideró simular ventilación adicional libre por ventanas y puertas que se abren cuando la temperatura interior supera el mínimo establecido de lunes a viernes entre las 8 a.m. y las 5 p.m.

Las tres escuelas estudiadas cuentan con el mismo modelo de aula. El aula fue simulada con los materiales de construcción típica de aula y con las propiedades que se definen en la Tabla 4. Para las simulaciones, los muros exteriores son considerados adiabáticos, excepto los que poseen ventanas (al exterior y hacia el pasillo).

\begin{tabular}{|c|c|c|c|c|c|}
\hline Componente & Material & $\begin{array}{l}\text { Valor U (W/ } \\
\text { m2K) }\end{array}$ & $\begin{array}{l}\text { Absortividad de } \\
\text { la superficie }\end{array}$ & $\begin{array}{l}\text { Valor U superficie } \\
\text { ext. (W/m2K) }\end{array}$ & $\begin{array}{l}\text { Factor de transmitancia } \\
\text { solar (G-value) }\end{array}$ \\
\hline Muro externo & $\begin{array}{l}\text { Columnas y muros modulares de } \\
\text { concreto prefabricado (Concreto } \\
\text { reforzado con } 1 \% \text { de acero) y espesor } \\
\text { de } 0.038 \mathrm{~m}\end{array}$ & 4,47 & 0,7 & 5,36 & - \\
\hline Cubierta & $\begin{array}{l}\text { Lámina exterior de acero esmaltado } \\
\text { blanco de } 0.44 \mathrm{~mm} \text {, aislante térmico de } \\
\text { EPS (Poliestireno expandido) de } 5 \mathrm{~mm} \\
\text { de espesor sobre estructura metálica } \\
\text { de hierro negro de } 15 \mathrm{~cm} \text { con calibre de } \\
1.5 \mathrm{~mm} \text { de espesor }\end{array}$ & 3,773 & 0,85 & 4,8 & - \\
\hline Cielo semi - expuesto & $\begin{array}{l}\text { Cielo suspendido de yeso de } 1 \mathrm{~cm} \text { de } \\
\text { espesor }\end{array}$ & 2,229 & - & - & - \\
\hline Piso interno & $\begin{array}{l}\text { Losa de } 10 \mathrm{~cm} \text { de espesor con baldosa } \\
\text { cerámica de } 13 \mathrm{~mm} \text { de espesor }\end{array}$ & 2,803 & 0,5 & - & - \\
\hline Acristalamiento & $\begin{array}{l}\text { Vidrio de } 3 \mathrm{~mm} \text { de espesor, claro, con } \\
\text { marquetería (herrajes) en aluminio }\end{array}$ & 5,894 & - & - & 0,7 \\
\hline
\end{tabular}

Tabla 4. Condiciones de borde utilizadas para simular los casos base de las tres localidades en estudio

Fuente: Elaboración propia

\section{Parámetros variables utilizados en las soluciones optimizadas}

Fueron considerados como parámetros variables para este estudio solamente los relativos a construcción y emplazamiento del edificio, ventilación, uso y operación de ventanas y control solar, con el fin de potencializar las estrategias de enfriamiento pasivo. Esto para que las variaciones puedan implementarse tanto en edificaciones existentes (sin incurrir en gastos elevados) como en nuevas construcciones bajo el mismo modelo constructivo propuesto por el Ministerio de Educación.

Los parámetros variables se basan en normas de diseño permitidas para el tipo de construcción y tienen la intención de observar cómo influye el cambio en la gestión de la ventilación, el control solar y el uso del sitio en su desempeño.

En total se simularon 640 variantes por escuela, 1920 simulaciones que definen combinaciones con mejor desempeño de acuerdo con cada ubicación. Los anteriores datos solo responden a la aplicación de las estrategias citadas anteriormente, sin pensar en variación de la envolvente. Posteriormente, se agregaron tres opciones más de validación (tomando en cuenta cambios en la cubierta y los muros) por cada variante con mejor desempeño en cada uno de los sitios estudiados. Lo anterior generó otras opciones de mejora mayores.

Estos rangos de variación se muestran en la Tabla 5, que resume todas las posibles combinaciones de variables para las simulaciones optimizadas. 


\begin{tabular}{|c|c|c|c|c|c|c|c|c|}
\hline Parámetro & \multicolumn{8}{|c|}{ Variantes - rangos } \\
\hline Orientación & $\begin{array}{l}\text { Norte } \\
\left(0^{\circ}\right)\end{array}$ & $\begin{array}{r}\text { Sur } \\
\left(180^{\circ}\right)\end{array}$ & $\begin{array}{l}\text { Este } \\
\left(90^{\circ}\right)\end{array}$ & $\begin{array}{l}\text { Oeste } \\
\left(270^{\circ}\right)\end{array}$ & $\begin{array}{l}\text { Noreste } \\
\left(45^{\circ}\right)\end{array}$ & $\begin{array}{l}\text { Noroeste } \\
\left(315^{\circ}\right)\end{array}$ & $\begin{array}{c}\text { Sureste } \\
\left(135^{\circ}\right)\end{array}$ & $\begin{array}{c}\text { Suroeste } \\
\left(225^{\circ}\right)\end{array}$ \\
\hline Ocupación & \multicolumn{4}{|c|}{ Media $(20 p)-0,39 p / m^{2}$} & \multicolumn{4}{|c|}{ Máxima $(34 p)-0,67 p / m^{2}$} \\
\hline $\begin{array}{l}\text { Cambio de aire e } \\
\text { infiltraciones }\end{array}$ & \multicolumn{8}{|c|}{ Dinámicas (calculadas por el programa de acuerdo a flujo, presiones del aire y cambios de temperatura) } \\
\hline $\begin{array}{l}\% \text { de área vidriada / } \\
\mathrm{m}^{2}\end{array}$ & \multicolumn{4}{|c|}{$20 \%$ (mínimo) } & \multicolumn{4}{|c|}{$30 \%$ (actual) } \\
\hline Espesor vidrio & \multicolumn{4}{|c|}{$3 \mathrm{~mm}$ (actual) } & \multicolumn{4}{|c|}{$6 \mathrm{~mm}$} \\
\hline Color vidrio & \multicolumn{4}{|c|}{ Claro-SHGC: 0,861} & \multicolumn{4}{|c|}{ Gris - SHGC: 0,602} \\
\hline Control solar & \multicolumn{4}{|c|}{$\begin{array}{l}\text { Aleros en fachada con ventanas a exterior } \\
\text { ( } 1 \text { metro - actual })\end{array}$} & \multicolumn{4}{|c|}{ Amplitud de alero a $1,20 \mathrm{~m}$} \\
\hline \multirow[b]{2}{*}{$\begin{array}{l}\text { Ventilación - } \\
\text { Operación de } \\
\text { ventanas }\end{array}$} & \multicolumn{8}{|c|}{ Ventilación cruzada diaria - Ventilación nocturna } \\
\hline & \multicolumn{2}{|c|}{$\begin{array}{l}\text { Ventanas } \\
\text { abiertas durante } \\
\text { lecciones }\end{array}$} & \multicolumn{2}{|c|}{$\begin{array}{c}\text { Ventanas abiertas } \\
\text { día y noche durante } \\
\text { lecciones (época seca) }\end{array}$} & \multicolumn{2}{|c|}{$\begin{array}{l}\text { Ventanas abiertas día y } \\
\text { noche todo el durante lecciones } \\
\text { (todo el año) }\end{array}$} & \multicolumn{2}{|c|}{$\begin{array}{l}\text { Ventanas abiertas día y noche todo e } \\
\text { año }\end{array}$} \\
\hline Apertura ventanas & \multicolumn{4}{|c|}{$\begin{array}{l}90 \% \text { de las ventanas abiertas al } 90 \%-95 \% \\
\text { de capacidad, } 10 \% \text { cerradas (Actual) }\end{array}$} & \multicolumn{4}{|c|}{$100 \%$ de las ventanas abiertas al $90 \%-95 \%$ de capacidad } \\
\hline Envolvente & \multicolumn{4}{|c|}{$\begin{array}{c}\text { Mantener materiales existentes en muros, } \\
\text { techumbre y pisos } \\
\text { Muros - Valor U: } 5,36 \mathrm{~W} / \mathrm{m}^{2} \mathrm{~K} \\
\text { Cubierta - Valor } \mathrm{U}: 3,77 \mathrm{~W} / \mathrm{m}^{2} \mathrm{~K} \\
\text { Piso - Valor U: } 2,803 \mathrm{~W} / \mathrm{m}^{2} \mathrm{~K}\end{array}$} & \multicolumn{4}{|c|}{$\begin{array}{l}\text { Cambio en Valor U de muro - Sistema Bloques de Hormigón } \\
\text { Muros - Valor U: } 2,274 \mathrm{~W} / \mathrm{m}^{2} \mathrm{~K}\end{array}$} \\
\hline $\begin{array}{c}\text { Manejo de } \\
\text { superficies cubiertas }\end{array}$ & \multicolumn{4}{|c|}{ Doble cubierta ventilada } & \multicolumn{4}{|c|}{ Doble cubierta ventilada con salida de aire superior (monitor) } \\
\hline
\end{tabular}

Tabla 5. Propiedades de los materiales que componen la envolvente y cerramientos del aula simulada.

Fuente: Elaboración propia.
Se utilizó un análisis probabilístico para identificar las posibilidades de un mejor rendimiento. El estudio se limita a las cinco (5) soluciones por ciudad que demostraron reducciones más altas. Las estrategias están encaminadas a evitar las ganancias térmicas y solares y promover la ventilación natural a un costo mínimo de inversión.

Las cinco (5) soluciones diferentes (Figura 4) fueron analizadas y comparadas con la situación actual (caso base):

Caso base. Configuración actual del aula escolar utilizando ventilación natural: cruzada diurna, acristalamiento de $30 \%$ del área construida con $80 \%$ de ventanas abiertas únicamente durante épocas de lecciones.

Caso A o Caso mejorado pasivo. Priorización de orientación de fachada vidriada con condiciones de ventilación predominante y control de ganancias por radiación. Ampliación de alero en fachada acristalada al exterior. Aprovechamiento de ventilación cruzada diurna y nocturna. Acristalamiento ajustado a $20 \%$ del área construida con aumento de superficie de muro. Ventanas abiertas todo el año durante lecciones con el $100 \%$ de área de ventanas con capacidad de apertura. Aprovechamiento de las propiedades del material con aumento de espesor de vidrio y tintura para reducción de ganancias solares.

Caso B o Caso mejorado + opción de masa térmica. Tomando como base el caso mejorado $A$, se incluyen cambios en la materialidad de la envolvente sin variación del volumen y aperturas. Utilización de sistema ampliamente utilizado de bloques de hormigón. El edificio gana masa térmica y se reduce el valor $\mathrm{U}$ de muros.

Caso $\mathrm{C}$ o Caso mejorado + doble techo con monitor: Tomando como base el caso mejorado A, se incluyen cambios en la superficie del techo con apertura para salida superior del aire y aleros superiores. 
Caso D o Caso mejorado + doble techo: Tomando como base el caso mejorado A, se incluyen cambios en la superficie del techo con doble cubierta ventilada sobre el área central de mayor incidencia.

Caso E o Caso mejorado + doble techo + masa térmica envolvente: Tomando como base el caso mejorado $A$, se incluyen cambios en la superficie del techo con doble cubierta ventilada sobre el área central de mayor incidencia. Se incluyen cambios en la materialidad de la envolvente utilizando bloques de hormigón. Ganancia de masa térmica y reducción de valor $\mathrm{U}$ de muros.

Se realizó un caso extra (Caso F) donde se usa de base el Caso E, pero se hace una simulación analizando la ocupación como un factor de peso en las condiciones internas del recinto. En el Caso $F$, se plantea la posibilidad de ajustes en los niveles de ocupación que permitan reducción de posibles condiciones de hacinamiento y mayores opciones de control de las ganancias internas.

Las variables utilizadas en los casos con un mejor rendimiento se componen de dos o más parámetros simultáneamente, lo que demuestra la necesidad de combinación de estrategias.
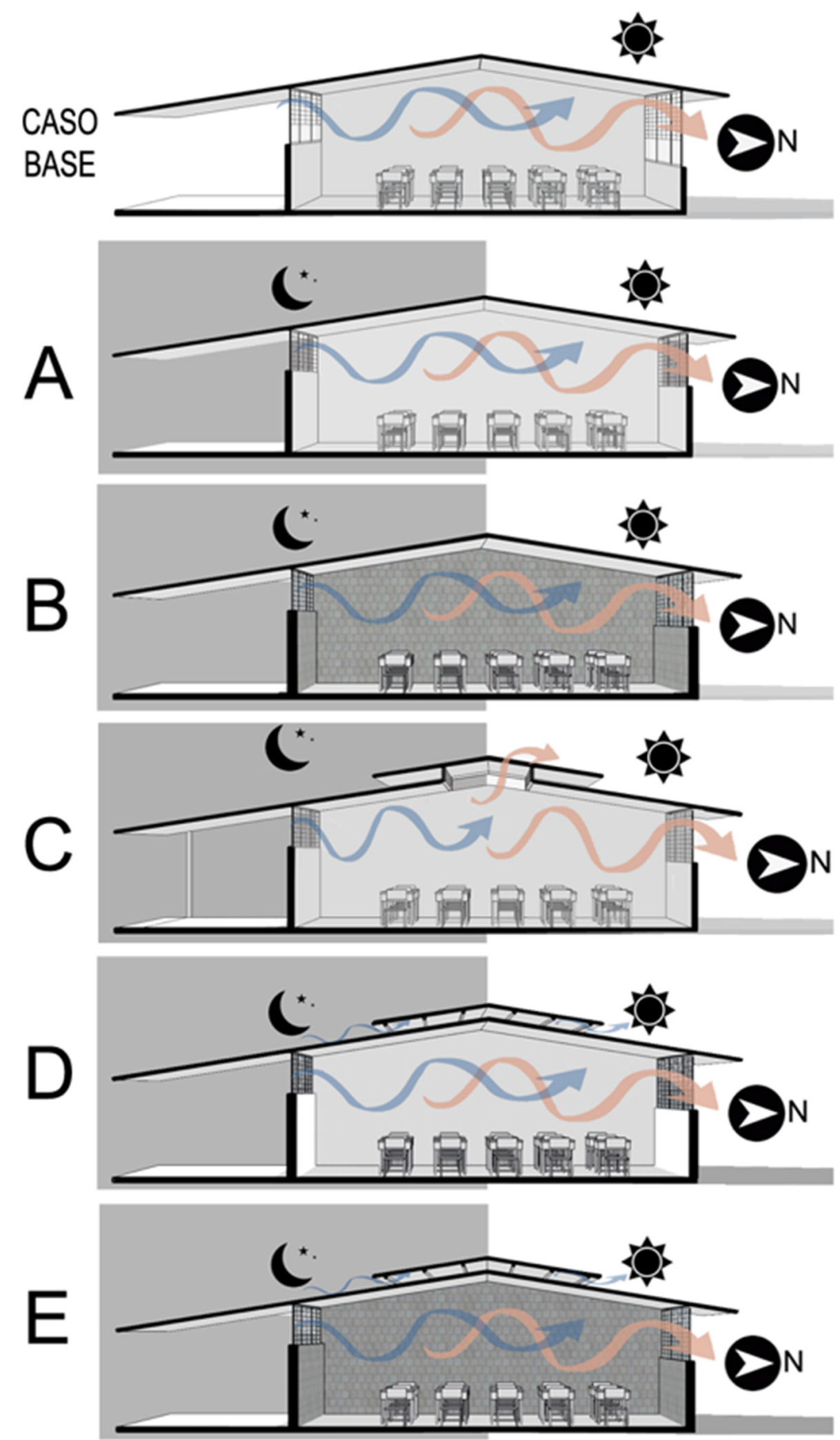

Figura 4. Diagramas de las combinaciones de estrategias con mejor desempeño a partir del caso base, aplicadas en los tres casos de estudio evaluados.

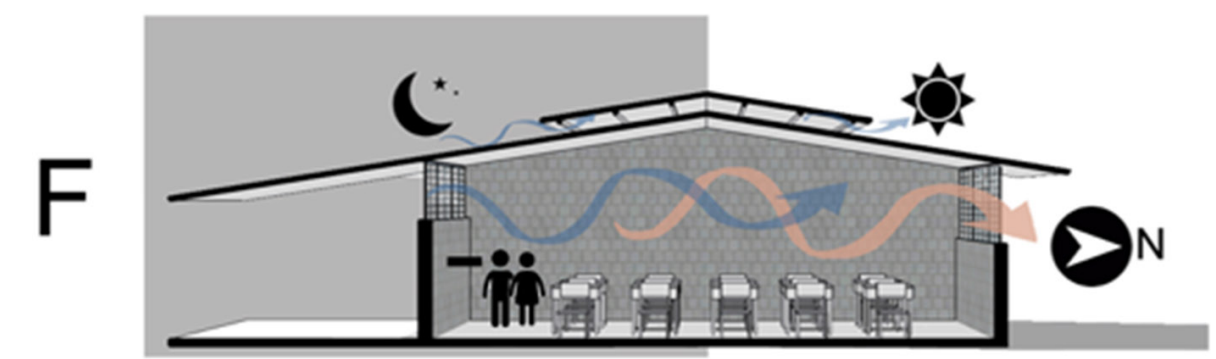




\section{Resultados de la simulación caso base y variaciones paramétricas}

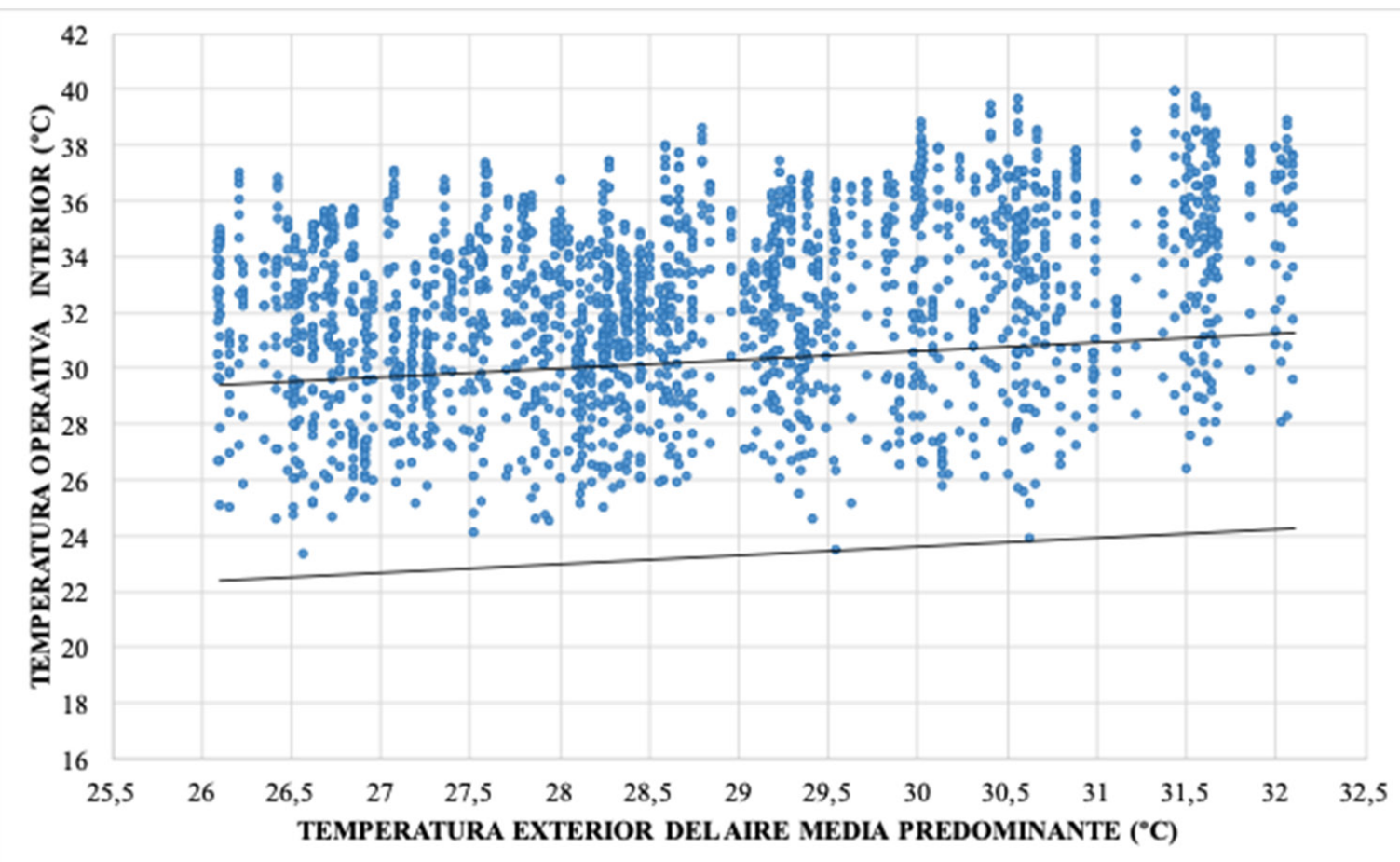

- CAso base bebedero

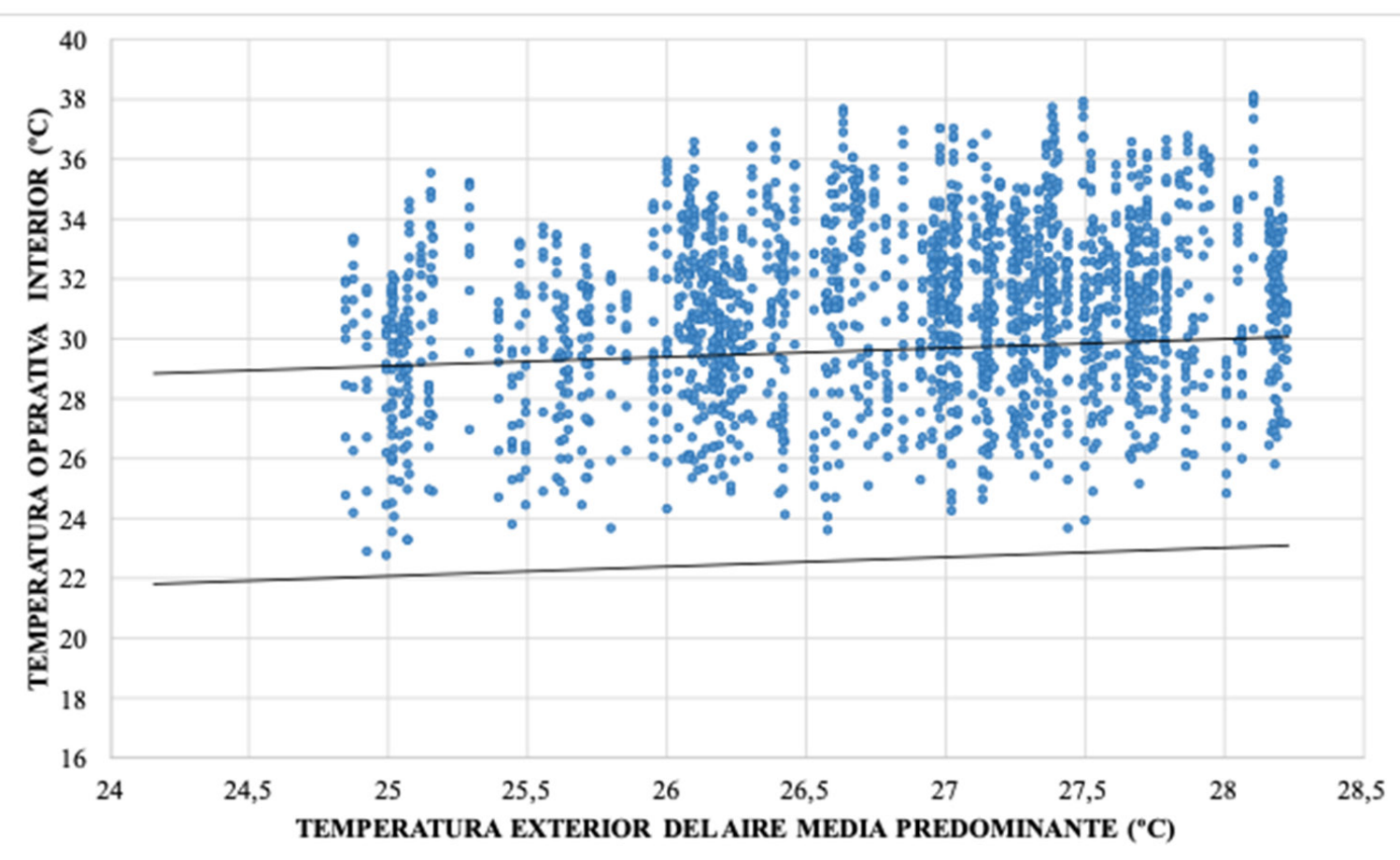

- CASo Base Paquita

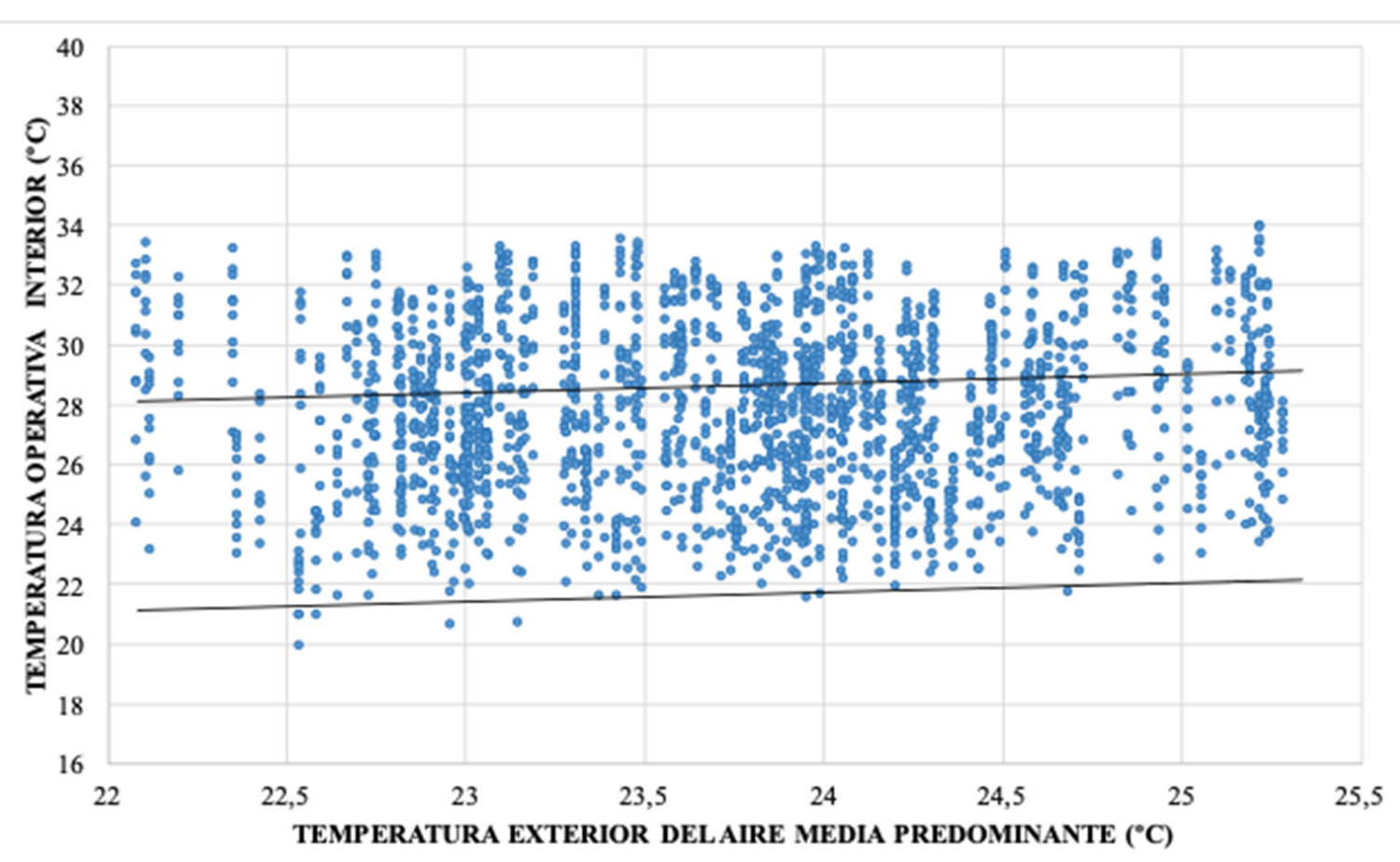

- CASo base buenos AIRES

Figura 5. Rango de temperaturas operativas aceptables por el $80 \%$ de los ocupantes para las tres localidades según el modelo de ASHRAE

55:2013 para espacios naturalmente ventilados.

Elaboración propia con respecto a datos calculados

en los casos de estudio.

\section{Desempeño energético}

Se simularon diferentes escenarios para cada localidad. Se realizó la evaluación de las ventajas conseguidas con respecto a la eficiencia energética para cada propuesta basada en la demanda anual.

Con respecto al caso base, se realizó un diagnóstico mediante la simulación, donde se fijaron los límites de confort en el software y se estableció un "setpoint" de $27^{\circ} \mathrm{C}$ como temperatura media de confort de acuerdo a los parámetros explicados anteriormente. Los casos fueron simulados con las condiciones de borde indicadas, aplicando el escenario de simulación con el sistema de enfriamiento encendido únicamente en los meses de funcionamiento de las aulas.

En los tres casos, entre marzo y junio, se presentan los mayores requerimientos de energía para el caso base, siendo marzo el mes más crítico para los casos de Bebedero y Veracruz y mayo para Paquita; sin embargo, para los meses más cálidos (época seca), se concentra en las tres localidades entre el $52 \%$ y el $56 \%$ del requerimiento energético anual, no muy lejano de las necesidades en época lluviosa. En las escuelas de Bebedero y Paquita, los valores se mantienen por encima de los $5000 \mathrm{kWh}(100 \mathrm{kWh} / \mathrm{m} 2 \mathrm{a})$, mientras que para Veracruz disminuye más de un $50 \%$ cercano a los $2000 \mathrm{kWh}$ $(39 \mathrm{kWh} / \mathrm{m} 2 \mathrm{a})$. Lo anterior debido a que las condiciones de Veracruz, en promedio anual, se mantienen más cercanas a los límites de confort que en los casos anteriores.

Se definieron 2040 horas operativas durante el año escolar, las cuales corresponden a un promedio de 200 días lectivos. Con esos datos, se analizaron paralelamente las horas de confort en cada caso base para comparar luego su desempeño con las mejoras propuestas en los escenarios de estudio. Los resultados en las tres localidades se resumen siguiendo el gráfico de temperaturas operativas horarias aceptables para $80 \%$ de los usuarios en espacios naturalmente ventilados (ASHRAE, 2013) como se muestra en la Figura 5 , donde se compara la temperatura operativa horaria con la temperatura media exterior predominante calculada para cada día en evaluación en los tres casos. 
Para el caso base de Bebedero, se encuentran 546 horas entre los límites de confort térmico para el $80 \%$ de los ocupantes y $26,76 \%$ del período de clases, para Paquita fueron 646 horas en confort y $31,7 \%$, y en el caso de Veracruz, Buenos Aires, un total de 1239 horas del año lectivo estuvieron en confort para un $60,74 \%$ del tiempo lectivo. Como es posible observar, la escuela de Bebedero presenta el mayor tiempo de lecciones fuera del rango de confort establecido (más del $70 \%$ del año) de acuerdo con la norma ASHRAE (2013).

Partiendo de los valores calculados para el caso base, se pretendió disminuir los promedios anuales e identificar así los mejores desempeños mediante los escenarios de mejoramiento, los cuales tendrían como objetivo una mejor respuesta con respecto a las condiciones térmicas interiores que afectan al usuario.

Los modelos de simulación optimizados se realizaron con ventilación mecánica y refrigeración por ventiladores en cielo, apoyados con el uso de la ventilación natural. Los mayores ahorros de energía se producen en épocas cálidas, sobre todo entre los meses de diciembre a abril.

Se eligió, en cada escuela, el caso con mejor desempeño con respecto a la aplicación de los parámetros relacionados con estrategias de ventilación y control solar, este caso se denominó "caso mejorado" (Caso A). Además, fueron aplicadas, con base en este escenario mejorado mediante las estrategias pasivas, otras posibles variaciones relacionadas con el cambio en la envolvente (muros y techumbre) con el fin de comparar el impacto que podían tener en este grupo con respecto al caso base.

La Tabla 6 resume las combinaciones con mejores resultados para las tres comunidades (Casos A) con menos energía requerida para el enfriamiento por metro cuadrado al año con la aplicación de las estrategias pasivas sin cambios en la envolvente (muros y techumbre).

Las mejoras obtenidas con relación al desempeño energético, utilizando estrategias pasivas no invasivas (Caso A), incluyen reducciones de entre $5 \%$ y $8 \%$ de los requerimientos energéticos para alcanzar el nivel de enfriamiento señalado. El caso Bebedero pasó de una demanda de $117,25 \mathrm{kWh} / \mathrm{m} 2 \mathrm{a}$ a $112 \mathrm{kWh} / \mathrm{m} 2 \mathrm{a}$, en la escuela de Paquita pasó de 105,2 kWh/m2a a $96 \mathrm{kWh} / \mathrm{m} 2$ a y en Veracruz de 40,58 kWh/m2a a $37,5 \mathrm{kWh} / \mathrm{m} 2 \mathrm{a}$ post-mejoramiento.

Como se observa, los casos de Bebedero y Paquita muestran la misma combinación de variables para lograr mejoras en el desempeño: se prioriza la orientación norte de la fachada vidriada sobre las otras y se demuestra un resultado exitoso al aplicar la ventilación nocturna como estrategia al menos durante el tiempo de lecciones tanto en estación seca como en lluviosa, combinada con ventilación cruzada diurna y aprovechando al máximo la capacidad de apertura de las ventanas. A estas estrategias del primer grupo (no invasivas) se unen otras que incluyen cambios de menor escala en condiciones y materialidad de la envolvente, como la reducción del área vidriada en un $10 \%$, eliminando acristalamiento fijo y colocando un módulo extra del sistema de muro prefabricado, extensión del alero a un metro y variar las características del vidrio con un aumento de espesor y con tintado gris.

En el caso mejorado para Veracruz, Buenos Aires, las condiciones varían en la orientación y el uso de la ventilación. Sobresale el resultado con una ubicación sureste de ventanas exteriores y sin el uso de ventilación nocturna como estrategia. El desempeño depende de los cambios en las condiciones de la envolvente para controlar la ganancia solar más que en las estrategias de disipación.

Se revisó la influencia por parámetros en la disminución de los requerimientos energéticos para enfriamiento por caso (Figura 6, Figura 7 y Figura 8), siendo el porcentaje de acristalamiento por área de construcción el factor que más influye en los tres casos, seguido por la operación y apertura de ventanas para Bebedero y Veracruz y la orientación de la fachada vidriada al exterior en Paquita. De manera independiente, estos factores lograron reducciones entre 3\% y 5\%. En contraposición, la orientación de fachadas vidriadas al este (Bebedero y Paquita) y al suroeste (Veracruz) son las variables que pueden afectar más el desempeño final al aumentar los requerimientos energéticos entre $1,5 \%$ y $5 \%$. 


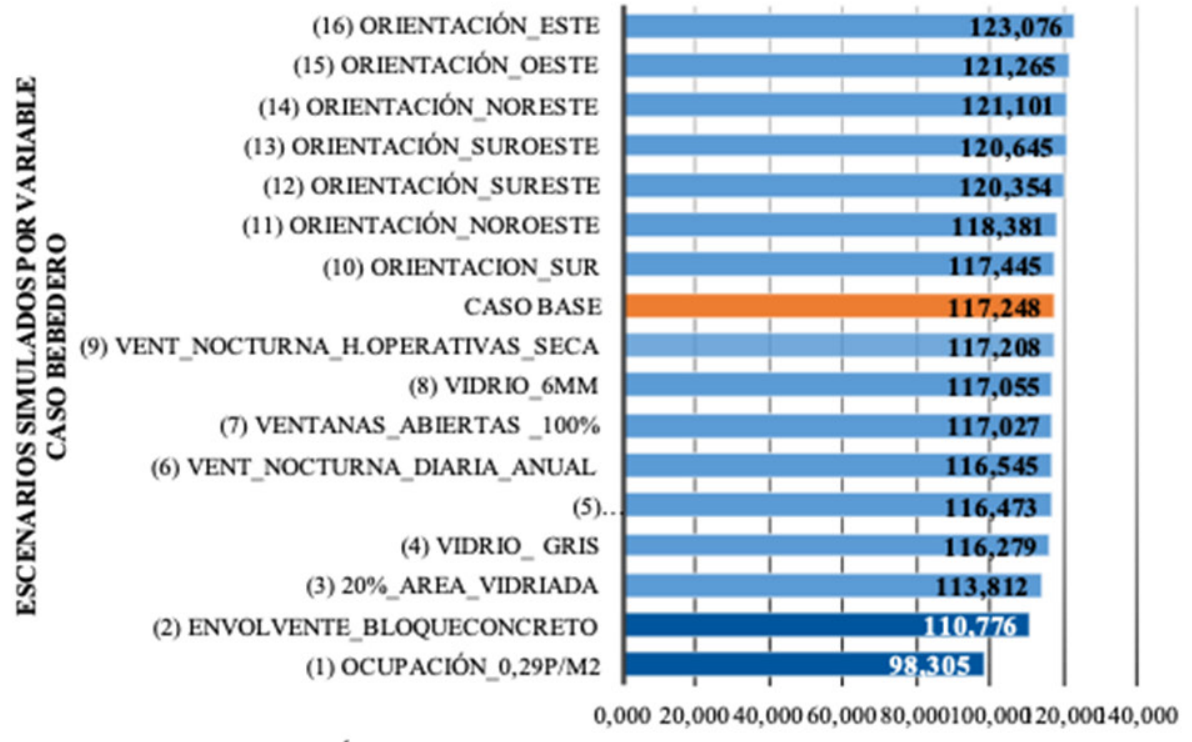

ENERGIA REQUERIDA PARA ENFRIAMIENTO (KWH/m2 a )

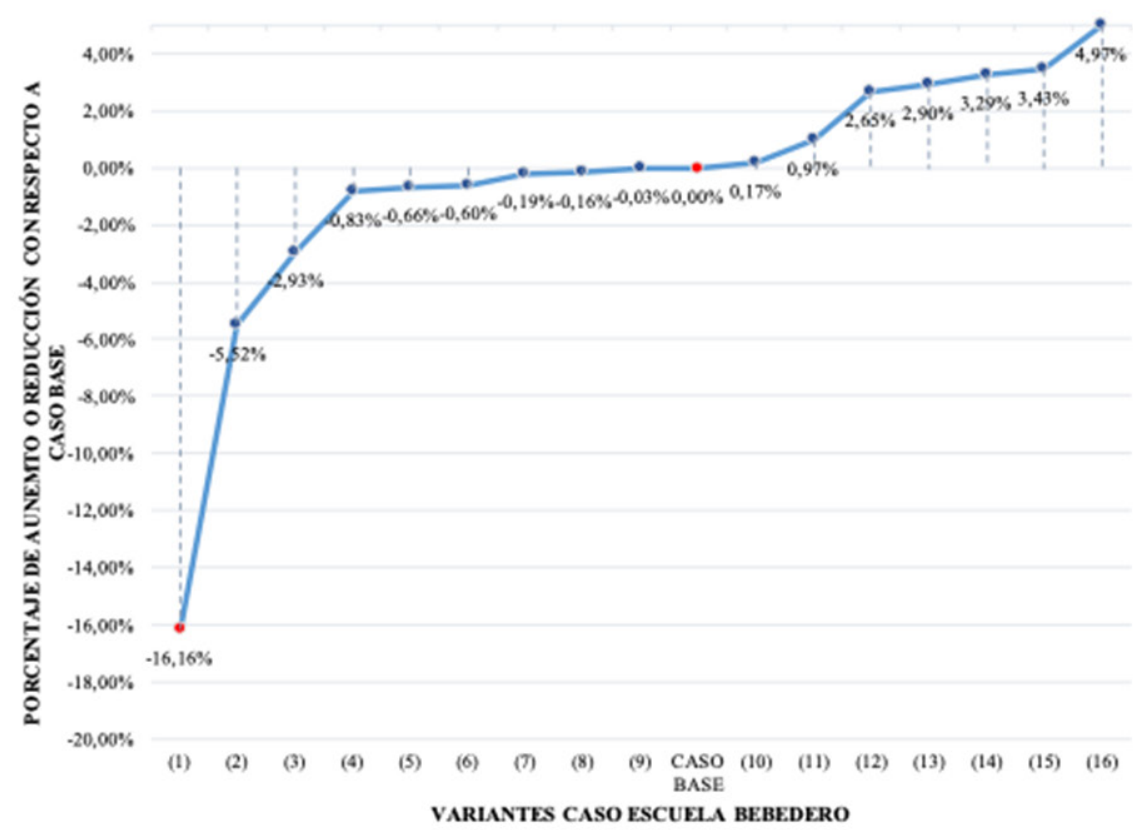

VARIANTES CASO ESCUELA BEBEDERO
Figura 6. Resultados de la influencia de distintas variables independientes en el

desempeño energético con respecto al caso base,

escuela Bebedero.

Fuente: Elaboración propia.

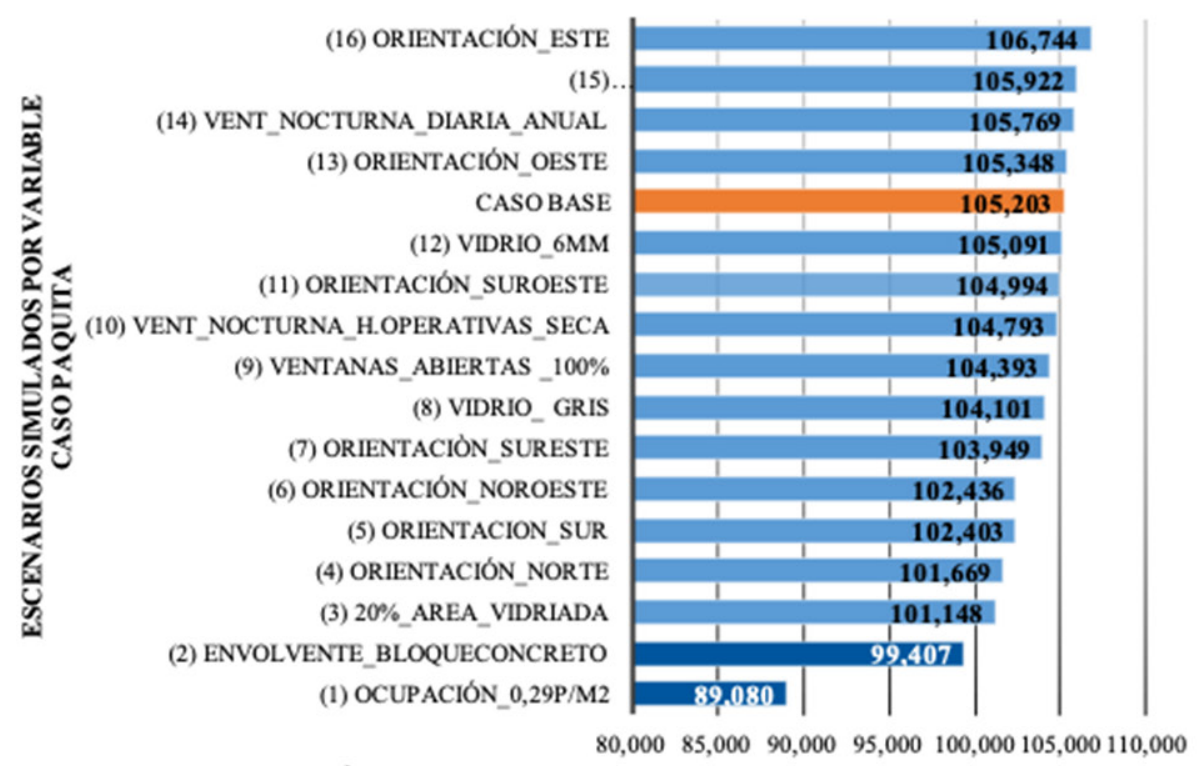

ENERGIA REQUERIDA PARA ENFRIAMIENTO (KWH/m2 a)

Figura 7. Resultados de la influencia de distintas variables independientes en el desempeño energético con respecto al caso base,

escuela Paquita.

Fuente: Elaboración propia.

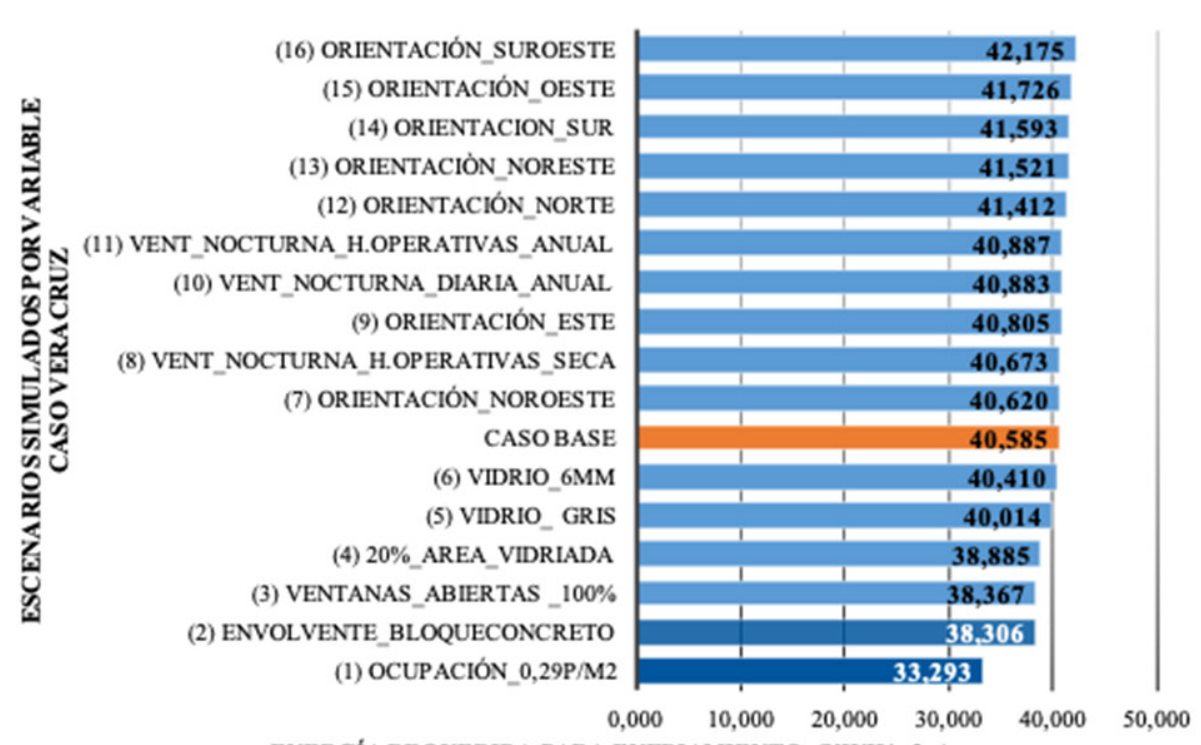

ENERGIA REQUERIDA PARA ENFRIAMIENTO (KWH/m2 a)

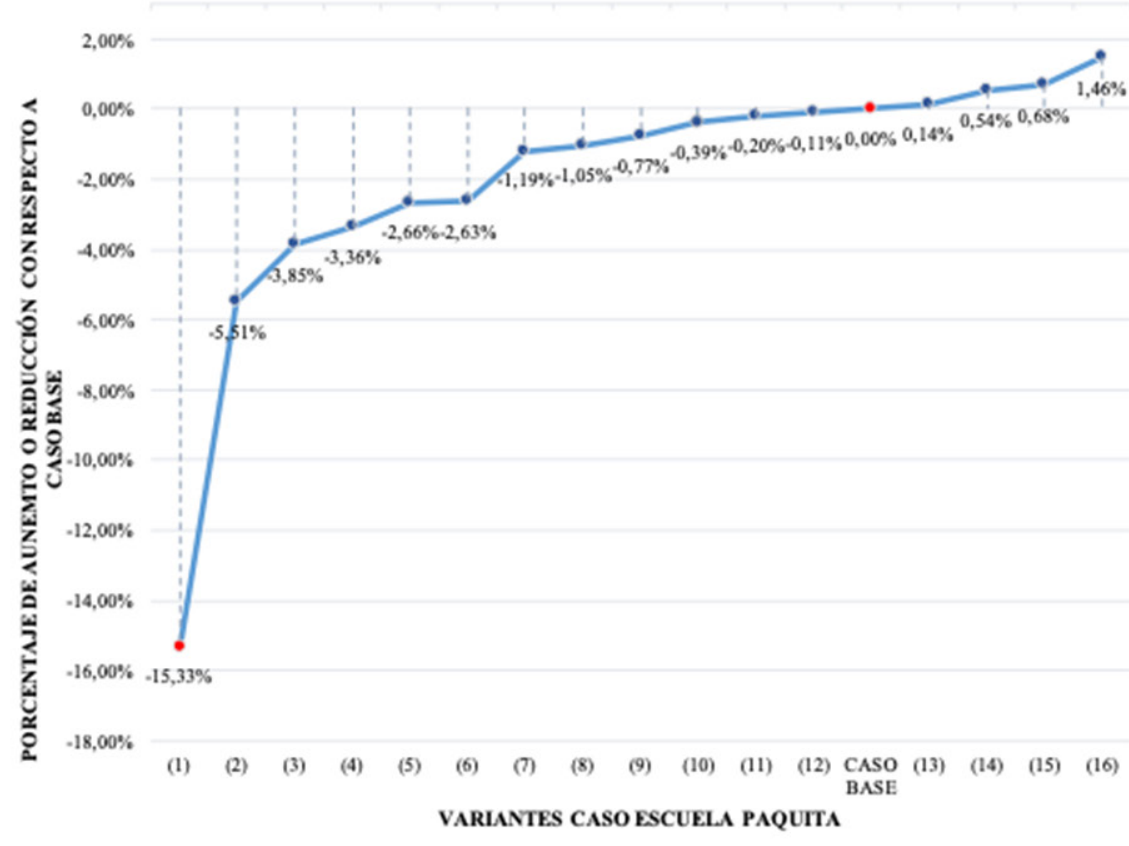

VARIANTES CASO ESCUELA PAQUTTA

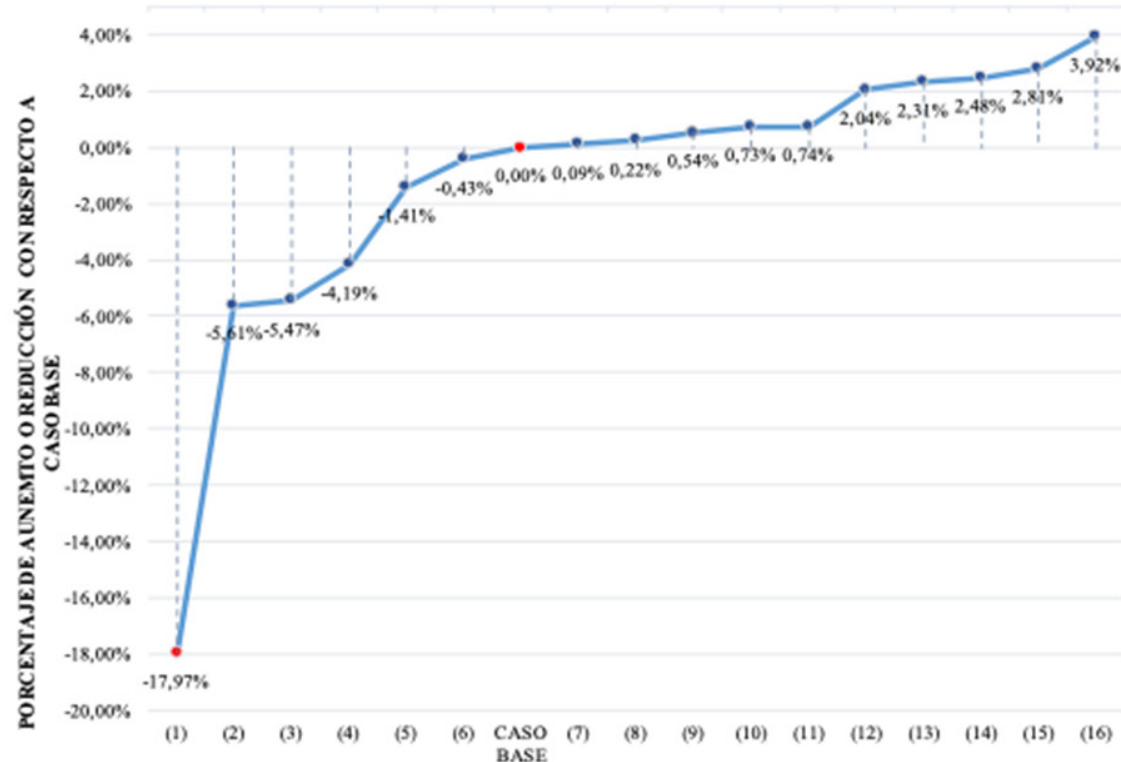

VARIANTES CASOESCUELA VERACRUZ 


\begin{tabular}{|c|c|c|c|}
\hline & $\begin{array}{l}\text { Caso mejorado } \\
\text { BEBEDERO }\end{array}$ & $\begin{array}{l}\text { Caso mejorado } \\
\text { PAQUITA }\end{array}$ & $\begin{array}{l}\text { Caso mejorado } \\
\text { VERACRUZ-BA }\end{array}$ \\
\hline Orientación de fachada vidriada exterior & Norte $\left(0^{\circ}\right)$ & Norte $\left(0^{\circ}\right)$ & Sureste $\left(225^{\circ}\right)$ \\
\hline Tipo de ventilación utilizada & Cruzada - Diurna y nocturna & Cruzada - Diurna y nocturna & Cruzada - Diurna \\
\hline Período de operación (ventilación) & Horas operativas/Anual & Horas operativas/Anual & Horas operativas/Anual \\
\hline Superficie vidriada total & $20 \%$ del área de aula & $20 \%$ del área de aula & $30 \%$ del área de aula \\
\hline $\begin{array}{l}\text { Porcentaje de superficie vidriada con posibilidad } \\
\text { de apertura }\end{array}$ & $100 \%$ & $100 \%$ & $100 \%$ \\
\hline Tipo de ventanas / vidriado & $\begin{array}{l}\text { Celosías } \\
\text { Vidrio simple-gris-6mm } \\
\text { Valor U:6,121W/m²-K } \\
\text { SHGC: } 0,567\end{array}$ & $\begin{array}{l}\text { Celosías } \\
\text { Vidrio simple-gris-6mm } \\
\text { Valor U:6,121W/m²-K } \\
\text { SHGC: } 0,567\end{array}$ & $\begin{array}{l}\text { Celosías } \\
\text { Vidrio simple-gris-6mm } \\
\text { Valor U: } 6,121 \mathrm{~W} / \mathrm{m}^{2}-\mathrm{K} \\
\text { SHGC: } 0,567\end{array}$ \\
\hline Protección solar en fachada vidriada exterior & Alero $-1 \mathrm{~m}$ & Alero $-1 \mathrm{~m}$ & Alero $-1 \mathrm{~m}$ \\
\hline Energía requerida para enfriamiento & $\begin{array}{l}5681,31 \mathrm{kWh} \\
112 \mathrm{kWh} / \mathrm{m}^{2} \mathrm{a}\end{array}$ & $\begin{array}{l}4897,06 \mathrm{kWh} \\
96 \mathrm{kWh} / \mathrm{m}^{2} \mathrm{a}\end{array}$ & $\begin{array}{l}1893,71 \mathrm{kWh} \\
37,5 \mathrm{kWh} / \mathrm{m}^{2} \mathrm{a}\end{array}$ \\
\hline Reducción con respecto al caso base & $4,05 \%$ & $7,82 \%$ & $7,60 \%$ \\
\hline
\end{tabular}

Tabla 6. Resumen de las combinaciones con mejores desempeños (CASO A) utilizando estrategias no invasivas para los tres casos en estudio según el análisis multifactorial aplicado. Fuente: Elaboración propia.
La mayoría de las medidas integrales aplicadas en el análisis multifactorial se benefician del control de cargas solares (manejo de aperturas) y la disipación de ganancias utilizando la ventilación; sin embargo, el factor ocupación (ganancias por cantidad de usuarios versus actividad) es un aspecto de difícil control y de donde se obtienen ganancias considerables, en algunos casos mayores que las ganancias solares a través de la envolvente y en donde se pueden obtener reducciones considerables.

Se realizó un ejercicio probando disminuir las cargas ocupacionales (a 0,29 personas/ m2-15 alumnos) y cambiando la envolvente a otro sistema constructivo (aumento de inercia térmica). Esas combinaciones se resumen en las opciones de los casos $B$ a F de la Figura 4. Al evaluar estas variables independientes, se aprecia que adicionan a la mejora energética entre 15\% y $17 \%$ con el manejo de la ocupación y entre $5 \%$ y $8 \%$ al utilizar la envolvente en mampostería (bloques de concreto). Ambas variables reducen una media mayor que la aplicación de cualquiera de las estrategias de disipación y control ya mencionadas, algunas trabajando en conjunto con las anteriores podrían lograr reducciones considerables entre un 30\% - 40\% (Ver Figura 9, Figura 10 y Figura 11).

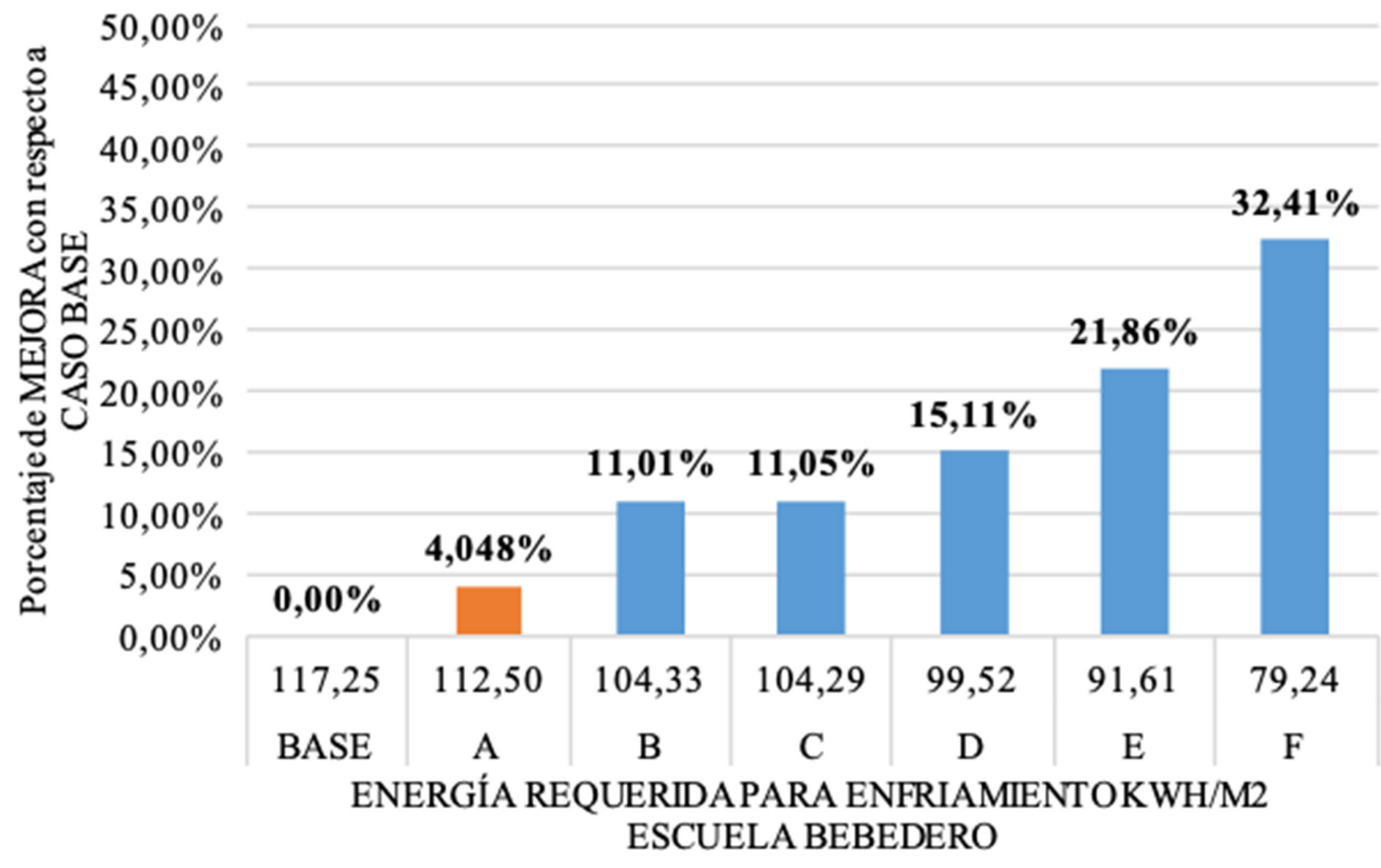


Figura 10. Porcentaje de mejora en el desempeño energético con respecto al caso base en las combinaciones totales aplicadas al caso escuela Paquita.

Fuente: Elaboración propia.
Figura 11. Porcentaje de mejora en el desempeño energético con respecto al caso base en las combinaciones totales aplicadas al caso escuela Veracruz, Buenos Aires. Fuente: Elaboración propia.
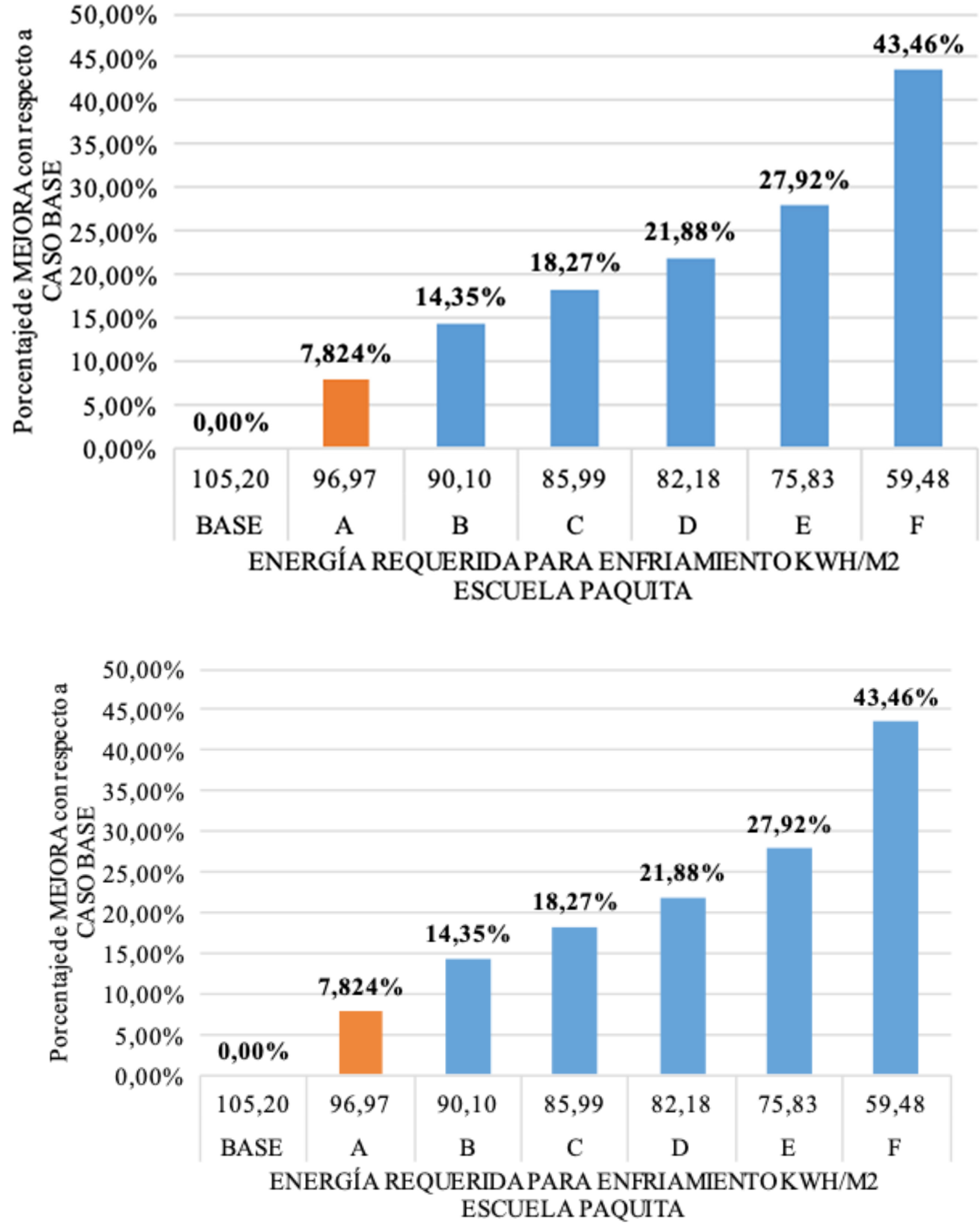

\section{Condiciones de confort}

Después de obtenidos los casos con mejor desempeño con respecto a la energía requerida para enfriamiento, se retoma la premisa de que estos son los casos que presentan temperaturas operativas más bajas en el interior del recinto. Se procedió a analizar el comportamiento térmico de estos con respecto a los límites de confort establecidos con el fin de determinar el nivel de reducción de la temperatura operativa y las afectaciones que pueda generar al usuario (con respecto a la disminución del riesgo de sobrecalentamiento).

Se realizó una comparación de la cantidad de horas lectivas (horas operativas de clase) dentro del rango de confort entre el caso base y el caso mejorado (Caso A), utilizando estrategias pasivas no invasivas en cada localidad.

Los resultados para el caso mejorado indican que, en la escuela de Bebedero, 599 horas están en el rango de confort establecido para el $80 \%$ de los ocupantes, $29,4 \%$ del tiempo y un aumento del $2,6 \%$ del tiempo con respecto al caso base. El período dentro del rango de confort para el caso de Paquita es de 752 horas que corresponden a $36,9 \%$ de las horas de clase, para un aumento de $5,2 \%$ del tiempo de lecciones. Por último, para la escuela de Veracruz, Buenos Aires, el aumento en horas de confort corresponde a $3,7 \%$ del tiempo lectivo, con 1315 horas dentro del rango de confort $64,5 \%$ del tiempo.

Los resultados en las escuelas de Bebedero y Paquita manifiestan que los usuarios pasan la mayoría de su tiempo de clases sobre el rango de confort establecido en esas localidades, aun considerando una disminución de entre $3 \%$ y $5 \%$ de horas operativas por encima de los límites de confort. Para marzo, uno de los meses críticos 
con mayores horas sobre el límite de confort, los períodos sobre ese límite pasaron de representar el $13 \%$ de las horas anuales que los estudiantes pasan en el centro educativo a porcentajes entre el $5 \%$ y el $8 \%$ dependiendo del centro educativo.

Los casos mejorados incluyen reducciones de entre $0,5^{\circ} \mathrm{C}$ y $1^{\circ} \mathrm{C}$ en 161 horas de clase (7,9\% del tiempo de lecciones) para Bebedero, 805 horas de clases (39,5\% del tiempo de lecciones) para el caso de Paquita y 186 horas $(9,12 \%$ de horas lectivas) para Veracruz. Solo en Paquita se incluye una reducción de más de $1^{\circ} \mathrm{C}$ en 234 horas de clase $(11,5 \%$ del tiempo lectivo).

Adicionalmente, como ejercicio, se aplicaron estrategias que incluyen cambios físicoconstructivos (modificaciones en la envolvente de muros y techumbre) que demostraron influir en mayor grado en el control de ganancias internas en los espacios estudiados. Estos aspectos corresponden a la transmitancia térmica de la envolvente, entendiéndose como la posibilidad de cambiar el material del aula por un sistema semi-prefabricado (bloques de concreto que reducen la transmitancia aproximadamente $50 \%$ y agrega masa térmica por medio del espesor de muro), se evaluó la respuesta de usar una doble cubierta ventilada (con y sin salida de aire) y otro factor como la ocupación, que es difícil de fijar en un valor determinado, pero que puede aproximarse en recintos que dependen de una matrícula como el caso de las escuelas.

Al aplicar estas variables sobre las características del caso mejorado, se obtiene un aumento combinado de entre $10 \%$ y $17 \%$ en las horas operativas anuales dentro del rango de confort; si se observa el nivel de mejora con respecto al caso base, los rangos aumentan a entre $15 \%$ y $25 \%$ de mejora. De estos aspectos, solo la reducción de la ocupación corresponde a un aumento de entre 3\% y $7 \%$ de horas en confort.

Los gráficos de las Figura 12, Figura 13 y Figura 14 muestran las variantes en los porcentajes de horas en confort al aplicar y combinar las diferentes variantes. El caso $\mathrm{A}$ corresponde a los cambios que incluyen únicamente aplicación de estrategias pasivas sin cambios en la envolvente. El caso $B$ toma en cuenta la variación en la masa térmica (espesor y transmitancia térmica del material). El caso $D$ incluye la variante de la envolvente de cubierta al agregar una doble techumbre ventilada. Por último, el caso $\mathrm{E}$ toma en cuenta la combinación de factores.

Figura 12. Variantes en los porcentajes de horas en confort en las diferentes combinaciones de estrategias aplicadas al caso escuela Bebedero.

Fuente: Elaboración propia

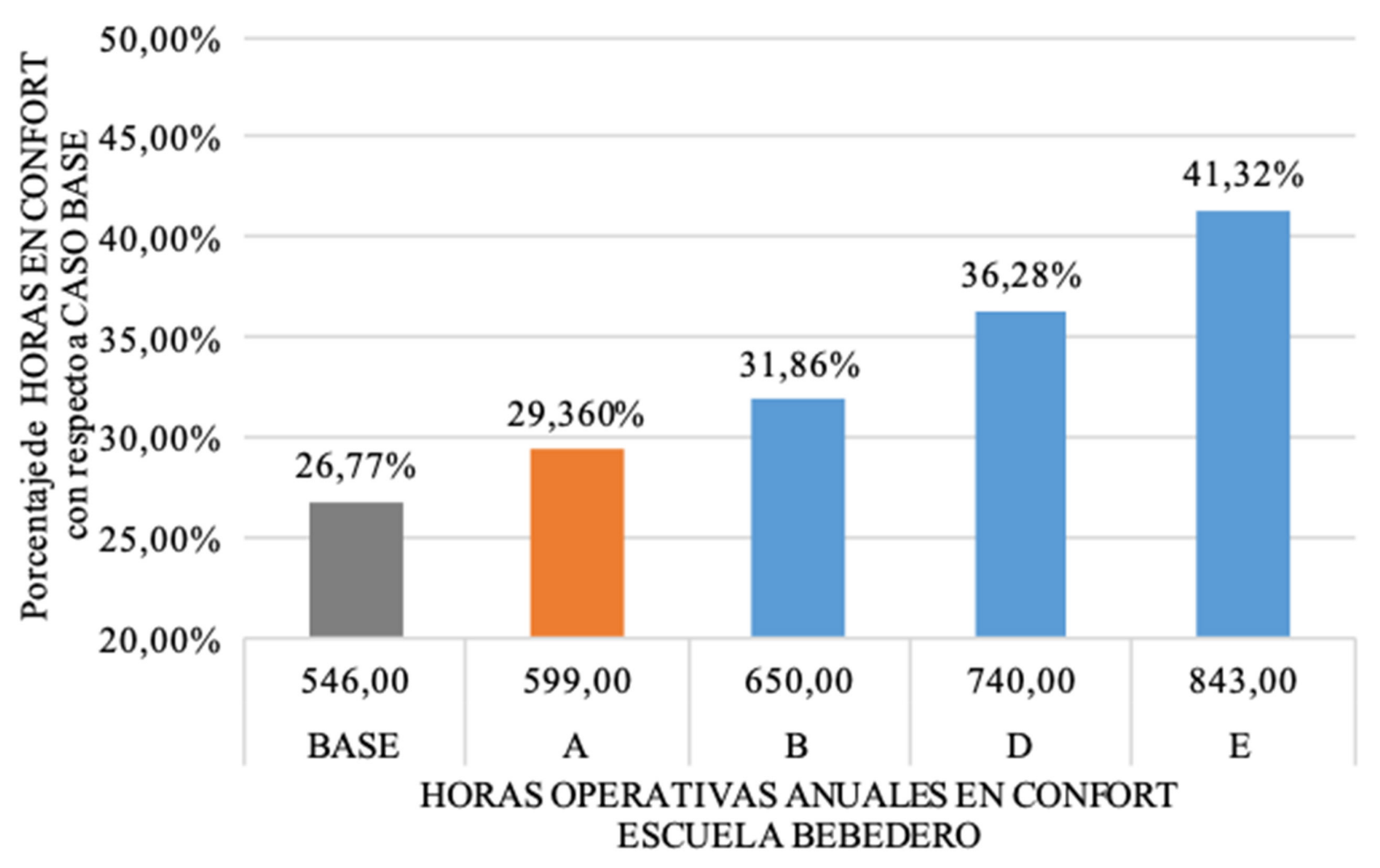


Figura 13. Variantes en los porcentajes de horas en confort en las diferentes combinaciones de estrategias aplicadas al caso escuela Paquita.

Fuente: Elaboración propia.
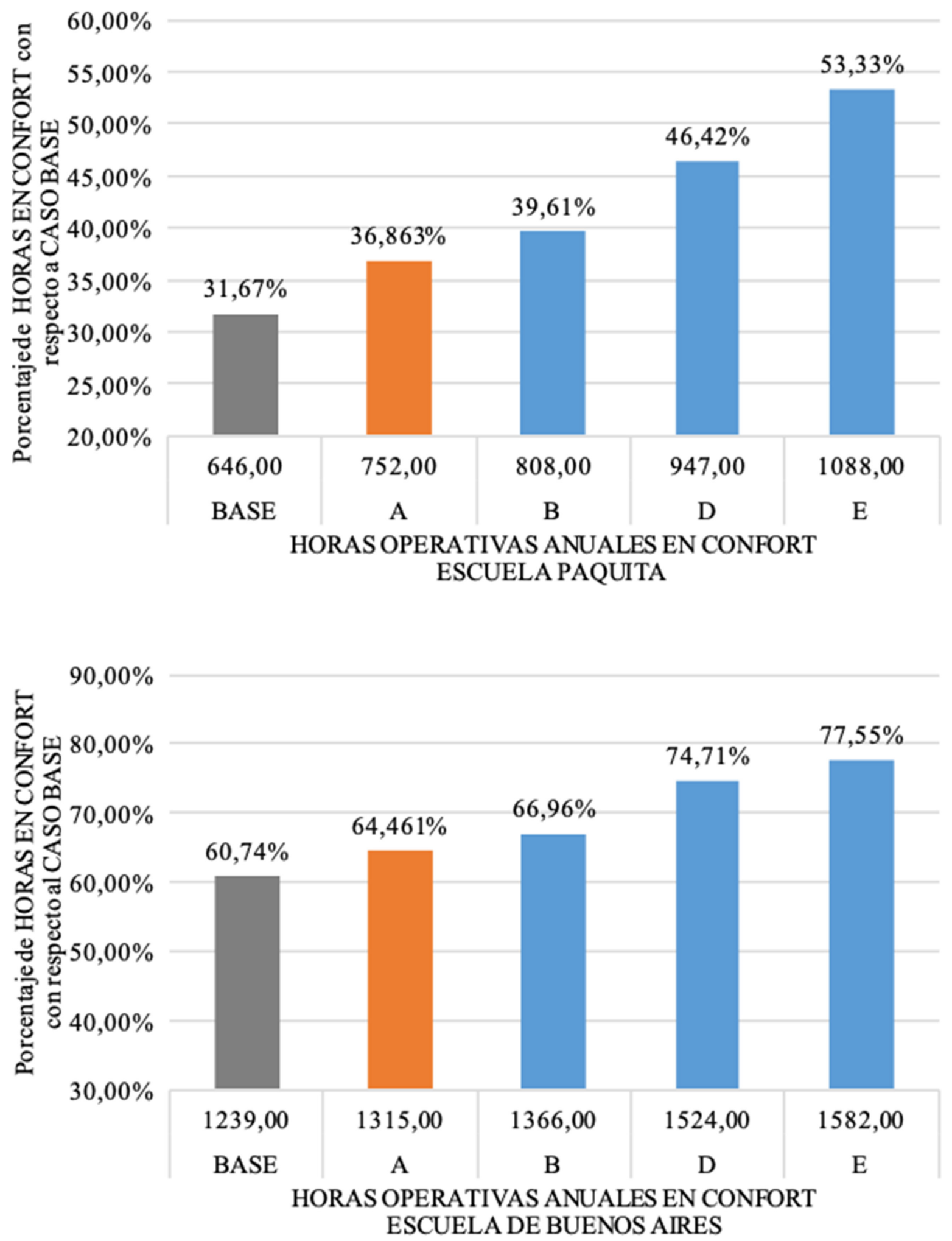

\section{Conclusiones}

El análisis térmico y energético de los tres (3) casos de estudios en la herramienta de simulación permitió considerar el desempeño de la tipología actual, además de lograr, a través de la comparación paramétrica, identificar las áreas que tienen un impacto positivo en las condiciones de confort interior y la eficiencia energética (reducción de la demanda de refrigeración).

Las simulaciones mostraron patrones conocidos dentro del diseño arquitectónico actual del aula, como la mejora del rendimiento de la orientación norte y el efecto de la altura y la superficie acristalada en la calidad térmica del espacio construido, al menos como estrategias no invasivas en el caso mejorado (Caso A).

La posibilidad de incrementar los cambios de aire a través de la ventilación natural y el control de las ganancias térmica ha demostrado ser clave para la desaceleración de la demanda en la refrigeración, por ejemplo, con el uso de ventiladores mecánicos 0 sistemas activos de acondicionamiento (HVAC), al menos en los casos estudiados.

Tanto a nivel de consumo energético como de confort térmico, en todos los casos los mejores resultados se lograron al combinar el uso de estrategias de enfriamiento pasivo no invasivas (caso mejorado o Caso A) con cambios del material y elementos de la envolvente (muros en mampostería y adición de una doble cubierta ventilada) junto con una reducción de la ocupación por metro cuadrado (Caso F). Si se toman en cuentan 
solamente los cambios realizables a nivel arquitectónico (Caso E) sin intervención del manejo de usuarios, igualmente se logran mejoras considerables de entre $21 \%$ y $29 \%$ con respecto al caso base.

Los cambios en la envolvente superior (cubierta) integrando dobles techumbres (Caso $C$ y Caso D) demuestran una influencia significativa en las mejoras de desempeño con respecto al uso de estrategias no invasivas al aumentar el porcentaje de mejora entre $8 \%$ y $17 \%$. Estas condiciones se deben a la influencia de la cubierta en la transmisión de ganancias solares en el interior de la edificación, tanto la ventilación de la cubierta como el doble material controlarían su impacto en el ambiente interno.

No obstante, hay que tomar en cuenta que la aplicación de estos factores (control de la ocupación y cambios en la envolvente) incluyen control de números de alumnos (matrícula anual y reducción de hacinamiento) e inversión en el desarrollo de propuestas con otros sistemas constructivos y su respectivo gasto económico para puesta en marcha, lo que puede darse como una condición futura por aplicar de parte de entidades gubernamentales.

A pesar de la valoración independiente de parámetros y la influencia de cada uno para lograr las mejoras energéticas evaluadas, es preciso tomar conciencia que el mejoramiento se debe propiciar utilizando las variables influyentes de manera integral. Las asociaciones de múltiples factores generaron los mejores desempeños en todos los casos.

La combinación o variables independientes que se emplearán (en caso de no poder aplicar el modelo óptimo para alguno de los casos) dependerán de la interacción de las opciones para determinar la estrategia por priorizar y el ahorro energético final a lograr.

La base de datos lograda del análisis realizado permite disponer de un margen de opciones de mejora que pueden combinarse dependiendo de las necesidades de implementación por parte de las entidades escolares en Costa Rica.

\section{Referencias bibliográficas}

ASHRAE. (2013). ANSI/ASHRAE Standard 55-2013: Thermal Environmental Conditions for Human Occupancy. American Society of Heating Refrigerating and AirConditioning Engineers.

Bordass, B., Cohen, R., Standeven, M. y Leaman, A. (2001). Assessing building performance in use 3: energy performance of the Probe buildings. Building Research \& Information, 29(2), 114-128. https://doi.org/10.1080/09613210010008036

Bravo, G. y González, E. (2001). Confort térmico en el trópico; hacia un estándar en viviendas naturalmente ventiladas. Información Tecnológica (Chile), 12(5), 169174.

Comisión Económica para América Latina y el Caribe. (2009). Situación y perspectivas de la eficiencia energética en América Latina y El Caribe. En Reunión Regional Intergubernamental: Eficiencia Energética en América Latina y el Caribe (p. 308).

Corgnati, S. P., Filippi, M. y Viazzo, S. (2007). Perception of the thermal environment in high school and university classrooms: Subjective preferences and thermal comfort. Building and Environment, 42(2), 951-959. https://doi.org/10.1016/j. buildenv.2005.10.027

de Dear, R. y Brager, G. S. (2001). The adaptive model of thermal comfort and energy conservation in the built environment. International Journal of Biometeorology, 45(2), 100-108. Recuperado de http://www.ncbi.nlm.nih.gov/pubmed/11513046

de Dear, R. (2006). The theory of thermal comfort in naturally ventilated indoor environments - "The Pleasure Principle." International Journal of Ventilation, 8(3), 243-250.

de Dear, R. (2011). Revisiting an old hypothesis of human thermal perception: alliesthesia. Building Research \& Information, 39(2), 108-117. https://doi.org/10.1080/096132 18.2011.552269 
Fanger, P. O. (1972). Thermal comfort: Analysis and applications in environmental engineering. Danish Technical Press. Recuperado de http://books.google.cl/ books/about/Thermal_comfort.html?id=S0FSAAAAMAAJ\&pgis=1

Givoni, B. (1994). Passive Low Energy Cooling of Buildings. John Wiley \& Sons. Recuperado de http://books.google.cl/books/about/Passive_Low_Energy_ Cooling_of_Buildings.html?id=rJsVoRw1geoC\&pgis=1

Givoni, B. (2011). Indoor temperature reduction by passive cooling systems. Solar Energy, 85(8), 1692-1726. https://doi.org/10.1016/j.solener.2009.10.003

Humphreys, M. a., Nicol, J. F. y Raja, I. a. (2007). Field Studies of Indoor Thermal Comfort and the Progress of the Adaptive Approach. Advances in Building Energy Research, 1(1), 55-88. https://doi.org/10.1080/17512549.2007.9687269

Liang, H.-H., Lin, T.-P. y Hwang, R.-L. (2012). Linking occupants' thermal perception and building thermal performance in naturally ventilated school buildings. Applied Energy, 94, 355-363. https://doi.org/10.1016/j.apenergy.2012.02.004

Lin, T.-P., de Dear, R., Matzarakis, A. y Hwang, R.-L. (2009). Prediction of thermal acceptability in hot-humid outdoor environments in Taiwan. En The seventh International Conference on Urban Climate,29 June - 3 July 2009, Yokohama, Japan (pp. 3-6).

Nitatwichit, C., Khunatorn, Y., Tantakitti, C., y Tippayawong, N. (2012). Simulation of flow and thermal comfort zones in a Thai state school. Journal of the Chinese Institute of Engineers, 35(1), 115-128. https://doi.org/10.1080/02533839.2012.625152

Prianto, E. y Depecker, P. (2003). Optimization of architectural design elements in tropical humid region with thermal comfort approach. Energy and Buildings, 35(3), 273-280. https://doi.org/10.1016/S0378-7788(02)00089-0

Programa Estado de la Nación en Desarrollo Humano Sostenible. (2013). Cuarto Informe Estado de la Educación (4 edición). San José: Editorama.

Rajapaksha, U. y Hyde, R. (2012). Barriers to and opportunities for advanced passive cooling in sub-tropical climates. Architectural Science Review, 55(1), 49-60. https://doi.org/10.1080/00038628.2011.641730

Rallapalli, H. (2010). A Comparison of EnergyPlus and eQUEST Whole Building Energy Simulation Results for a Medium Sized Office Building. Recuperado de http://129.219.247.59/attachments/56303/content/Rallapalli_asu_0010N_10220. pdf

Roaf, S., Nicol, F. y de Dear, R. (2013). The wicked problem of designing for comfort in a rapidly changing world. Architectural Science Review, 56(1), 1-3.

Sosa Griffin, M.E. y Siem, G. (2004). Manual de Diseñopara Edificaciones Enérgicamente Eficientes en el Trópico (Primera Ed). Caracas: IDEC.

Walter, K. (2000). La educación en Centroamérica: Reflexiones en torno a sus problemas y su potencial [No. CA 2020: Documento de trabajo \# 10].

Wargocki, P. y Wyon, D. P. (2007). The effects of moderately raised classroom temperatures and classroom ventilation rate on the performance of schoolwork by children. HVAC\&R Research, 13(2), 193-220. 